ESAIM: M2AN 50 (2016) 635-650

DOI: $10.1051 / \mathrm{m} 2 \mathrm{an} / 2015051$
ESAIM: Mathematical Modelling and Numerical Analysis

www.esaim-m2an.org

\title{
BRIDGING THE HYBRID HIGH-ORDER AND HYBRIDIZABLE DISCONTINUOUS GALERKIN METHODS
}

\author{
Bernardo Cockburn ${ }^{1}$, Daniele A. Di Pietro ${ }^{2}$ and Alexandre Ern ${ }^{3}$
}

\begin{abstract}
We build a bridge between the hybrid high-order (HHO) and the hybridizable discontinuous Galerkin (HDG) methods in the setting of a model diffusion problem. First, we briefly recall the construction of HHO methods and derive some new variants. Then, by casting the HHO method in mixed form, we identify the numerical flux so that the HHO method can be compared to HDG methods. In turn, the incorporation of the HHO method into the HDG framework brings up new, efficient choices of the local spaces and a new, subtle construction of the numerical flux ensuring optimal orders of convergence on meshes made of general shape-regular polyhedral elements. Numerical experiments comparing two of these methods are shown.
\end{abstract}

Mathematics Subject Classification. 65N30, 65N08.

Received February 10, 2015. Revised July 4, 2015.

Published online May 23, 2016.

\section{INTRODUCTION}

The Hybrid High-Order (HHO) method has been recently introduced in [16] in the context of quasiincompressible linear elasticity. We consider here its application (studied in $[17,18]$ ) to the numerical approximation of the model problem: find $u \in H_{0}^{1}(\Omega)$ such that

$$
\int_{\Omega} \kappa \nabla u \cdot \nabla v=\int_{\Omega} f v \quad \forall v \in H_{0}^{1}(\Omega)
$$

where $\Omega \subset \mathbb{R}^{d}$ is a bounded, connected polyhedral domain and $\boldsymbol{\kappa}$ a bounded, symmetric, uniformly positivedefinite matrix-valued function. We assume, additionally, that $\boldsymbol{\kappa}$ is piecewise Lipschitz on a partition $P_{\Omega}$ of $\Omega$ into polyhedra. An extension to more general singularly-perturbed advection-diffusion problems can be found in [13].

The HHO method supports general polyhedral meshes and delivers an arbitrary-order accurate approximation using face-based discrete unknowns that are polynomials of degree at most $k$ on each face. The method encompasses the case $k=0$, for which connections exist with the Hybrid Finite Volume method of [23] (see also [21]),

\footnotetext{
Keywords and phrases. Hybridizable discontinuous Galerkin, hybrid high-order, variable diffusion problems.

1 School of Mathematics, University of Minnesota, Minneapolis, USA. cockburn@math.umn.edu

2 University of Montpellier, Institut Montpelliérain Alexander Grothendieck, 34095 Montpellier, France.

daniele.di-pietro@umontpellier.fr

3 University Paris-Est, CERMICS (ENPC), 77455 Marne-la-Vallée cedex 2, France, ern@cermics.enpc.fr
} 
the Compatible Discrete Operator framework of [4], and the Mimetic Finite Difference methods of [5, 6, 27]; $c f$. also the recent monograph [3]. The HHO method is derived in terms of a primal formulation, and is designed from two key ingredients:

(i) a potential reconstruction in each mesh cell and

(ii) a face-based stabilization consistent with the high-order provided by the reconstruction.

The design relies on intermediate cell-based discrete unknowns in addition to the face-based ones (hence, the term hybrid). The cell-based unknowns can be eliminated by static condensation, as already pointed out in $[16,18]$ (without giving details). In this work, we derive some new variants of the HHO method resulting from the choice of cell-based unknowns, allowing us to draw some connections with the recently derived High-Order Mimetic (HOM) method introduced in [30] for general $\boldsymbol{\kappa}$ and analyzed for $\boldsymbol{\kappa}=\boldsymbol{I}_{d}$ in [2]. We also describe in more details the static condensation since this operation is particularly important in practice.

Our second important task is to recast the HHO method into an equivalent mixed formulation. This allows us to identify the corresponding conservative numerical flux and compare to Hybridizable Discontinuous Galerkin (HDG) methods within the general framework introduced in [9]. Our approach in identifying the flux is different from the one proposed in [15], where local conservativity was obtained for the HHO method by means of auxiliary local Neumann problems. The HDG methods were originally devised as discrete versions of a characterization of the exact solution in terms of solutions of local problems globally matched through transmission conditions. Following ideas from [12] in the framework of the Stokes equations, we show how the approximate solution provided by the HHO method can also be characterized as the solution of local problems which are then matched by a single global equation. We then provide an interpretation of such equation as a discrete version of a transmission condition. This allows us to uncover the numerical trace of the flux for the HHO method and then fit the HHO method in the HDG framework. We show that both the local spaces and numerical trace of the flux are novel, distinctive choices which enrich the family of HDG methods. In particular, the spaces for the flux are much smaller than the ones previously known, and the stabilization function displays a rich structure that allows for optimal convergence of both the potential $u$ and its flux $\boldsymbol{q}:=-\kappa \nabla u$ on quite general meshes composed of polyhedral cells. We then use the HDG framework to compare several methods including the LDG-H methods [9] (a subclass of the DG methods proposed in [7]), the HDG methods introduced in [29] and analyzed in [31], and some new methods. We end by comparing the actual performance of a couple of these methods.

The organization of the paper is as follows. In Section 2, we recall the definition of the HHO method, its main convergence properties, and present in more details the static condensation procedure. We also present some new variants of the HHO method resulting from the choice of cell-based unknowns. In Section 3, we rewrite the method in the numerical-trace formulation. In Section 4, we use this rewriting to compare it to other HDG methods theoretically as well as numerically. We end in Section 5 with some concluding remarks.

\section{THE HYBRID HIGH-ORDER METHOD}

In this section, we recall the definition of the HHO method and its convergence properties. The exposition introduces a generalization of the original method proposed in $[16,18]$ which allows us to cover two variants corresponding to different choices of the intermediate cell-based unknowns.

\subsection{Notation}

Denote by $\mathcal{H} \subset \mathbb{R}_{*}^{+}$a countable set of meshsizes having 0 as its unique accumulation point. Following ([14], Chap. 4), we consider $h$-refined mesh sequences $\left(\mathcal{T}_{h}\right)_{h \in \mathcal{H}}$ where, for all $h \in \mathcal{H}, \mathcal{T}_{h}$ is a finite collection of nonempty

disjoint open polyhedral elements $T$ of boundary $\partial T$ composed of planar faces, such that $\bar{\Omega}=\bigcup_{T \in \mathcal{T}_{h}} \bar{T}$ and $h=\max _{T \in \mathcal{T}_{h}} h_{T}$ with $h_{T}$ standing for the diameter of the element $T$.

A face $F$ is defined as a hyperplanar closed connected subset of $\bar{\Omega}$ with positive $(d-1)$-dimensional Hausdorff measure and such that (i) either there exist $T_{1}, T_{2} \in \mathcal{T}_{h}$ such that $F \subset \partial T_{1} \cap \partial T_{2}$ and $F$ is called an interface 
or (ii) there exists $T \in \mathcal{T}_{h}$ such that $F \subset \partial T \cap \partial \Omega$ and $F$ is called a boundary face. Interfaces are collected in the set $\mathcal{F}_{h}^{\mathrm{i}}$, boundary faces in $\mathcal{F}_{h}^{\mathrm{b}}$, and we let $\mathcal{F}_{h}:=\mathcal{F}_{h}^{\mathrm{i}} \cup \mathcal{F}_{h}^{\mathrm{b}}$. The diameter of a face $F \in \mathcal{F}_{h}$ is denoted by $h_{F}$. For all $T \in \mathcal{T}_{h}, \mathcal{F}_{T}:=\left\{F \in \mathcal{F}_{h} \mid F \subset \partial T\right\}$ denotes the set of faces $\subset \partial T$ and, for all $F \in \mathcal{F}_{T}, \boldsymbol{n}_{T F}$ is the unit normal to $F$ pointing out of $T$. We also define the piecewise constant vector-valued field $\boldsymbol{n}_{T}$ on $\partial T$ such that $\boldsymbol{n}_{T \mid F}=\boldsymbol{n}_{T F}$ for all $F \in \mathcal{F}_{T}$.

We assume that, for all $h \in \mathcal{H}, \mathcal{T}_{h}$ admits a matching simplicial submesh $\mathfrak{T}_{h}$ such that any cell and any face in $\mathfrak{T}_{h}$ belongs to only one cell and face of $\mathcal{T}_{h}$, respectively, and there exists a real number $\varrho>0$ independent of $h$ such that (i) for all simplex $S \in \mathfrak{T}_{h}$ of diameter $h_{S}$ and inradius $r_{S}$, $\varrho h_{S} \leqslant r_{S}$ and (ii) for all element $T \in \mathcal{T}_{h}$, and all simplex $S \in \mathfrak{T}_{h}$ such that $S \subset T, \varrho h_{T} \leqslant h_{S}$. These assumptions allow one to derive local trace and inverse inequalities (cf., e.g., [14], Chap. 1) as well as optimal polynomial approximation properties [22]. We emphasize that the simplicial submesh is a theoretical tool which is not used in the constructions underlying both HHO and HDG methods. Additionally, we suppose that, for all $h \in \mathcal{H}, \mathcal{T}_{h}$ is compatible with $\kappa$, meaning that, for all $T \in \mathcal{T}_{h}$, there exists a unique subdomain $\Omega_{i}$ in $P_{\Omega}$ containing $T$. As a result, jumps of $\kappa$ can occur at interfaces but not inside elements.

Let $l \geqslant 0$. For all $T \in \mathcal{T}_{h}, \mathbb{P}_{d}^{l}(T)$ is composed of the $d$-variate polynomial functions of degree $\leqslant l$ restricted to $T$, while, for all $F \in \mathcal{F}_{h}, \mathbb{P}_{d-1}^{l}(F)$ is composed of the $(d-1)$-variate polynomial functions of degree $\leqslant l$ restricted to $F$. For all $T \in \mathcal{T}_{h}$, we denote by $\pi_{T}^{l}$ the $L^{2}$-orthogonal projector onto $\mathbb{P}_{d}^{l}(T)$.

In what follows, we often abbreviate $a \lesssim b$ the inequality $a \leqslant C b$ for positive real numbers $a$ and $b$ and a generic constant $C$ which can depend on $\varrho, d, \kappa$ and the considered polynomial degree, but is independent of $h$.

For a subset $X \subset \bar{\Omega}$, we denote by $(\cdot, \cdot)_{X}$ and $\|\cdot\|_{X}$ the usual $L^{2}(X)$-inner product and norm, with the convention that we omit the index if $X=\Omega$. The same notation is used for the vector space $L^{2}(X)^{d}$.

\subsection{Local construction}

Let two integers $k \geqslant 0$ and $l \in\{k-1, k, k+1\}$ be fixed; in the case $k=0$, we only consider for simplicity that $l \in\{k, k+1\}$ and refer to Remark 2.1 for the modifications required when $k=0$ and $l=k-1$. The choice $l=k$ corresponds to the original HHO method introduced in [16], whereas the choice $l=k-1$ essentially leads (up to an equivalent choice of the stabilization) to the HOM method introduced in [30], see Section 2.4 below. The choice $l=k+1$ yields, in turn, a novel method (see also Rem. 2.2 below). For all $T \in \mathcal{T}_{h}$, we define the local space of discrete potential unknowns as follows:

$$
\underline{\mathrm{U}}_{T}^{k, l}:=\mathrm{U}_{T}^{l} \times \mathrm{U}_{\partial T}^{k}, \quad \mathrm{U}_{T}^{l}:=\mathbb{P}_{d}^{l}(T), \quad \mathrm{U}_{\partial T}^{k}:=\underset{F \in \mathcal{F}_{T}}{\chi} \mathbb{P}_{d-1}^{k}(F) .
$$

Elements of the local space $\underline{\mathrm{U}}_{T}^{k, l}$ are underlined, and a generic element of $\underline{\mathrm{U}}_{T}^{k, l}$ is denoted by $\underline{\mathrm{v}}_{T}=\left(\mathrm{v}_{T},\left(\mathrm{v}_{F}\right)_{F \in \mathcal{F}_{T}}\right)$ or, in more compact form, as $\underline{\mathbf{v}}_{T}=\left(\mathrm{v}_{T}, \mathrm{v}_{\partial T}\right)$, where $\mathrm{v}_{\partial T}$ is the piecewise polynomial function such that $\mathrm{v}_{\partial T \mid F}=\mathrm{v}_{F}$ for all $F \in \mathcal{F}_{T}$. We can define a higher-order potential reconstruction operator $p_{T}^{k+1}: \underline{\mathrm{U}}_{T}^{k, l} \rightarrow \mathbb{P}_{d}^{k+1}(T)$ as follows: for a given $\underline{\mathbf{v}}_{T} \in \underline{\mathrm{U}}_{T}^{k, l}, p_{T}^{k+1} \underline{\mathbf{v}}_{T}$ solves the Neumann problem

$$
\left(\boldsymbol{\kappa} \nabla p_{T}^{k+1} \underline{\mathbf{v}}_{T}, \boldsymbol{\nabla} w\right)_{T}=\left(\boldsymbol{\kappa} \boldsymbol{\nabla} \mathrm{v}_{T}, \boldsymbol{\nabla} w\right)_{T}+\left(\mathrm{v}_{\partial T}-\mathrm{v}_{T}, \boldsymbol{\kappa} \boldsymbol{\nabla} w \cdot \boldsymbol{n}_{T}\right)_{\partial T} \quad \forall w \in \mathbb{P}_{d}^{k+1}(T),
$$

with closure condition given by $\left(p_{T}^{k+1} \underline{\mathbf{v}}_{T}, 1\right)_{T}=\left(\mathrm{v}_{T}, 1\right)_{T}$. We next introduce the local bilinear form $a_{T}: \underline{\mathrm{U}}_{T}^{k, l} \times$ $\underline{\mathrm{U}}_{T}^{k, l} \rightarrow \mathbb{R}$ such that

$$
a_{T}\left(\underline{\mathbf{w}}_{T}, \underline{\mathbf{v}}_{T}\right):=\left(\kappa \nabla p_{T}^{k+1} \underline{\mathbf{w}}_{T}, \boldsymbol{\nabla} p_{T}^{k+1} \underline{\mathbf{v}}_{T}\right)_{T}+s_{T}\left(\underline{\mathbf{w}}_{T}, \underline{\mathbf{v}}_{T}\right),
$$

where the stabilizing bilinear form $s_{T}: \underline{\cup}_{T}^{k, l} \times \underline{\cup}_{T}^{k, l} \rightarrow \mathbb{R}$ is such that

$$
s_{T}\left(\underline{\mathbf{w}}_{T}, \underline{\mathbf{v}}_{T}\right):=\left(\tau_{\partial T} \pi_{\partial T}^{k}\left(P_{T}^{k+1, l} \underline{\mathbf{w}}_{T}-\mathrm{w}_{\partial T}\right), \pi_{\partial T}^{k}\left(P_{T}^{k+1, l} \underline{\mathbf{v}}_{T}-\mathrm{v}_{\partial T}\right)\right)_{\partial T},
$$


where $\tau_{\partial T}$ is a piecewise constant function on $\partial T$ such that $\tau_{\partial T \mid F}=\frac{\kappa_{T F}}{h_{F}}$ for all $F \in \mathcal{F}_{T}$ with $\kappa_{T F}=\boldsymbol{n}_{F} \cdot \boldsymbol{\kappa}_{\mid T} \cdot \boldsymbol{n}_{F}$, $\pi_{\partial T}^{k}$ is the $L^{2}$-orthogonal projector on $\bigcup_{\partial T}^{k}$, and $P_{T}^{k+1, l}: \underline{\cup}_{T}^{k, l} \rightarrow \mathbb{P}_{d}^{k+1}(T)$ is obtained adding to the function $\mathrm{v}_{T}$ a high-order correction inferred from $p_{T}^{k+1}$ :

$$
P_{T}^{k+1, l} \underline{\mathbf{v}}_{T}:=\mathbf{v}_{T}+\left(p_{T}^{k+1} \underline{\mathbf{v}}_{T}-\pi_{T}^{l} p_{T}^{k+1} \underline{\mathbf{v}}_{T}\right) .
$$

Let $\underline{\underline{L}}_{T}^{k, l}: H^{1}(T) \rightarrow \underline{\mathrm{U}}_{T}^{k, l}$ be the reduction map such that, for all $T \in \mathcal{T}_{h}$ and all $v \in H^{1}(T)$,

$$
\underline{\underline{I}}_{T}^{k, l} v=\left(\pi_{T}^{l} v, \pi_{\partial T}^{k} v\right) .
$$

The potential reconstruction operator $p_{T}^{k+1}$ and the bilinear form $s_{T}$ are conceived so that they satisfy the following two key properties:

(i) Stability. There is a real number $\eta>0$ independent of $T$ and of $h$ such that, for all $\underline{\mathbf{v}}_{T} \in \underline{\mathbf{U}}_{T}^{k, l}$,

$$
\eta\left\|\underline{\mathbf{v}}_{T}\right\|_{a, T}^{2} \leqslant\left\|\boldsymbol{\kappa} \nabla \mathbf{v}_{T}\right\|_{L^{2}(T)^{d}}^{2}+j_{T}\left(\underline{\mathbf{v}}_{T}, \underline{\mathbf{v}}_{T}\right) \leqslant \eta^{-1}\left\|\underline{\mathbf{v}}_{T}\right\|_{a, T}^{2},
$$

with local energy seminorm such that $\left\|\underline{\mathbf{v}}_{T}\right\|_{a, T}^{2}:=a_{T}\left(\underline{\mathrm{v}}_{T}, \underline{\mathrm{v}}_{T}\right)$ and boundary-jump bilinear form $j_{T}: \underline{\mathrm{U}}_{T}^{k, l} \times \underline{\mathrm{U}}_{T}^{k, l} \rightarrow \mathbb{R}$ defined as

$$
j_{T}\left(\underline{\mathrm{w}}_{T}, \underline{\mathrm{v}}_{T}\right):=\left(\tau_{\partial T}\left(\mathrm{w}_{T}-\mathrm{w}_{\partial T}\right), \mathrm{v}_{T}-\mathrm{v}_{\partial T}\right)_{\partial T} .
$$

The dependence of $\eta$ on $\boldsymbol{\kappa}$ is specified in ([17], Lem. 3.1).

(ii) Approximation. For all $v \in H^{k+2}(T)$,

$$
\left\{\left\|\boldsymbol{\nabla}\left(v-p_{T}^{k+1} \underline{\underline{1}}_{T}^{k, l} v\right)\right\|_{T}^{2}+s_{T}\left(\underline{\underline{L}}_{T}^{k, l} v, \underline{\mathbf{l}}_{T}^{k, l} v\right)\right\}^{1 / 2} \lesssim h_{T}^{k+1}\|v\|_{H^{k+2}(T)} .
$$

Unlike the bilinear form $s_{T}$, the bilinear form $j_{T}$ only satisfies, for all $v \in H^{k+1}(T)$,

$$
j_{T}\left(\underline{l}_{T}^{k, l} v, \underline{\mathbf{l}}_{T}^{k, l} v\right)^{1 / 2} \lesssim h^{k}\|v\|_{H^{k+1}(T)} .
$$

For this reason, it has not been used in the formulation of the method (2.12). Some remarks are of order.

Remark 2.1 $(k=0$ and $l=k-1)$.

In this case, the following conventions are adopted: (i) in (2.1), element unknowns are not needed in the construction; (ii) the closure condition for problem (2.2) is modified by prescribing that $\left(p_{T}^{k+1} \underline{\mathbf{v}}_{T}, 1\right)_{T}=\sum_{F \in \mathcal{F}_{T}} \omega_{T F} \mathbf{v}_{F}$ with weights $\left(\omega_{T F}\right)_{F \in \mathcal{F}_{T}}$ defined as in ([30], Appendix A). (iii) in (2.5), it is understood that $\pi_{T}^{l} p_{T}^{k+1} \underline{\mathbf{v}}_{T}=0$. This case is closely related (up to an equivalent choice of the stabilization) to Hybrid Finite Volumes [23]. Recalling the equivalence result proved in [21] for Mixed [20] and Hybrid Finite Volumes and Mimetic Finite Differences $[5,6,27]$, the HHO methods with $k=0$ and $l=-1$ or $l=0$ lead in fact to the same family of methods (up to equivalent choices of stabilization).

Remark $2.2(l=k+1)$.

In this case, we have $\pi_{T}^{k+1} p_{T}^{k+1} \underline{\mathbf{v}}_{T}=p_{T}^{k+1} \underline{\mathbf{v}}_{T}$ since $p_{T}^{k+1} \underline{\mathbf{v}}_{T} \in \mathbb{P}_{d}^{k+1}(T)$, so that $P_{T}^{k+1, k+1} \underline{\mathbf{v}}_{T}=\mathbf{v}_{T}$, and the stabilizing bilinear form $s_{T}$ simply writes

$$
s_{T}\left(\underline{\mathrm{w}}_{T}, \underline{\mathrm{v}}_{T}\right):=\left(\tau_{\partial T} \pi_{\partial T}^{k}\left(\mathrm{w}_{T}-\mathrm{w}_{\partial T}\right), \pi_{\partial T}^{k}\left(\mathrm{v}_{T}-\mathrm{v}_{\partial T}\right)\right)_{\partial T} .
$$

A similar stabilization was suggested in ([29], Rem. 1.2.4) in the context of HDG methods; cf. Table 1 for further details. 


\subsection{Definition of the method and error estimates}

We define the global spaces

$$
\underline{\mathrm{U}}_{h}^{k, l}:=\mathrm{U}_{\mathcal{T}_{h}}^{l} \times \mathrm{U}_{\mathcal{F}_{h}}^{k}, \quad \bigcup_{\mathcal{T}_{h}}^{l}:=\underset{T \in \mathcal{T}_{h}}{\chi} \mathbb{P}_{d}^{l}(T), \quad \bigcup_{\mathcal{F}_{h}}^{k}:=\underset{F \in \mathcal{F}_{h}}{\chi} \mathbb{P}_{d-1}^{k}(F),
$$

and we introduce a subspace of $\underline{\underline{U}}_{h}^{k, l}$ with strongly enforced boundary conditions:

$$
\underline{\mathrm{U}}_{h, 0}^{k, l}:=\mathrm{U}_{\mathcal{T}_{h}}^{l} \times \mathrm{U}_{\mathcal{F}_{h}, 0}^{k}, \quad \mathrm{U}_{\mathcal{F}_{h}, 0}^{k}:=\left\{\mathrm{v}_{\mathcal{F}_{h}} \in \mathrm{U}_{\mathcal{F}_{h}}^{k} \mid \mathrm{v}_{F} \equiv 0 \forall F \in \mathcal{F}_{h}^{\mathrm{b}}\right\} .
$$

For an element $T \in \mathcal{T}_{h}$ and a function $\underline{\mathrm{v}}_{h}=\left(\mathrm{v}_{\mathcal{T}_{h}}, \mathrm{v}_{\mathcal{F}_{h}}\right) \in \underline{\mathrm{U}}_{h}^{k, l}$, we denote by $\underline{\mathrm{v}}_{T}:=\left(\mathrm{v}_{T}, \mathrm{v}_{\partial T}\right)$ its restriction to $\underline{\mathrm{U}}_{T}^{k, l}$. The global bilinear form $a_{h}: \underline{\mathrm{U}}_{h}^{k, l} \times \underline{\mathrm{U}}_{h}^{k, l}$ is assembled elementwise from the local contributions (2.3):

$$
a_{h}\left(\underline{\mathrm{w}}_{h}, \underline{\mathrm{v}}_{h}\right):=\sum_{T \in \mathcal{T}_{h}} a_{T}\left(\underline{\mathrm{w}}_{T}, \underline{\mathrm{v}}_{T}\right) \text {. }
$$

The discrete problem reads: find $\underline{\mathrm{u}}_{h} \in \underline{\mathrm{U}}_{h, 0}^{k, l}$ such that

$$
a_{h}\left(\underline{\mathrm{u}}_{h}, \underline{\mathrm{v}}_{h}\right)=\sum_{T \in \mathcal{T}_{h}}\left(f, \mathrm{v}_{T}\right)_{T} \quad \forall \underline{\mathrm{v}}_{h} \in \underline{\mathbf{U}}_{h, 0}^{k, l} .
$$

We next recall the a priori error estimates obtained in [18] for the case $l=k$. Minor variations in the proofs yield analogous results for the cases $l=k \pm 1$. Our estimates are stated in terms of quantities we define next. We denote by $\underline{l}_{h}^{k, l}: H^{1}(\Omega) \rightarrow \underline{\mathrm{U}}_{h}^{k, l}$ the operator whose restriction to $H^{1}(T)$ is $\underline{\mathbf{l}}_{T}^{k, l}(c f$. (2.6)), and we define by $p_{h}^{k+1} \underline{\mathbf{u}}_{h}$ the function whose restriction to $T$ is $p_{T}^{k+1} \underline{\mathbf{u}}_{T}$. We also define on $\underline{\underline{U}}_{h, 0}^{k, l}$ the global energy norm $\left\|\underline{\mathrm{v}}_{h}\right\|_{a, h}^{2}:=\sum_{T \in \mathcal{T}_{h}}\left\|\underline{\mathbf{v}}_{T}\right\|_{a, T}^{2}$ (the fact that $\|\cdot\|_{a, h}$ defines a norm on $\underline{\mathrm{U}}_{h, 0}^{k, l}$ follows from the strong enforcement of boundary conditions). We are now ready to state the result. For simplicity, we do not explicitate the dependence of the constants on the diffusion tensor; see [17] for a more precise result.

Theorem 2.3 (Error estimate for HHO). Let $u \in H_{0}^{1}(\Omega)$ and $\underline{\underline{u}}_{h} \in \underline{\mathrm{U}}_{h, 0}^{k, l}$ denote the unique solutions to (1.1) and (2.12), respectively, and assume the additional regularity $u \in H^{k+2}(\Omega)$. Then, there exists a real number $C>0$ depending on $\varrho, k, d$, and $\boldsymbol{\kappa}$, but independent of $h$, such that

$$
\max \left(\left\|p_{h}^{k+1} \underline{\mathrm{u}}_{h}-u\right\|,\left\|\mathrm{u}_{h}-\pi_{h}^{l} u\right\|,\left\|\underline{\mathrm{u}}_{h}-\underline{\underline{l}}_{h}^{k, l} u\right\|_{a, h}\right) \leqslant C h^{k+1}\|u\|_{H^{k+2}(\Omega)},
$$

where $\mathbf{u}_{h}$ is the broken polynomial function such that $\mathbf{u}_{h \mid T}=\mathbf{u}_{T}$ for all $T \in \mathcal{T}_{h}$ and $\pi_{h}^{l}$ the $L^{2}$-orthogonal projector onto the space of broken polynomials of total degree $\leqslant l$. Additionally, if elliptic regularity holds, and further assuming $f \in H^{1}(\Omega)$ if $k=0$, we have the improved $L^{2}$-error estimate

$$
\max \left(\left\|p_{h}^{k+1} \underline{\mathbf{u}}_{h}-u\right\|,\left\|\mathbf{u}_{h}-\pi_{h}^{l} u\right\|\right) \leqslant C h^{k+2}\left(\|u\|_{H^{k+2}(\Omega)}+\|f\|_{H^{k+\delta}(\Omega)}\right),
$$

with $\delta=1$ if $k=0$ and $\delta=0$ if $k \geqslant 1$.

\subsection{Link with HOM}

It is possible to devise a (minor) extension of the setting considered in [2] for the convergence analysis of the HOM method in the case $\boldsymbol{\kappa}=\boldsymbol{I}_{d}$. This extension, which consists in considering two, possibly different, polynomial degrees in the virtual space of [2], allows us to bridge the actual HOM method to HHO with $l=k-1$ where the difference appears in an equivalent choice of stabilization, and to offer the possibility of devising new variants of HOM which can be bridged to the HHO method for $l \in\{k, k+1\}$. Another benefit of this extension is to provide a convergence analysis of HOM in the variable-diffusion case. 
Assume $\boldsymbol{\kappa}=\boldsymbol{I}_{d}$ and define, for all $T \in \mathcal{T}_{h}$, the local space

$$
V_{T}^{k, l}:=\left\{\varphi \in H^{1}(T) \mid \nabla \varphi_{\mid \partial T} \cdot \boldsymbol{n}_{T} \in \mathrm{U}_{\partial T}^{k} \text { and } \triangle \varphi \in \mathbb{P}_{d}^{l}(T)\right\} .
$$

The difference with respect to [2] consists in the choice of the space for $\Delta \varphi$, which now accounts for all the possible values for the polynomial degree $l$. It is useful to note, at this point, that the explicit expression of functions in $V_{T}^{k, l}$ is, in general, not available.

Consider the map $\Phi_{T}: \underline{\mathrm{U}}_{T}^{k, l} \rightarrow V_{T}^{k, l}$ defined such that, for all $\underline{\mathrm{v}}_{T} \in \underline{\mathrm{U}}_{T}^{k, l}, \varphi:=\Phi_{T}\left(\underline{\mathrm{v}}_{T}\right)$ solves

$$
\begin{aligned}
\triangle \varphi & =\mathrm{v}_{T}-|T|_{d}^{-1}\left\{\left(\mathrm{v}_{T}, 1\right)_{T}-\left(\mathrm{v}_{\partial T}, 1\right)_{\partial T}\right\} & & \text { in } T, \\
\boldsymbol{\nabla} \varphi \mid \partial T \cdot \boldsymbol{n}_{T} & =\mathrm{v}_{\partial T} & & \text { on } \partial T, \\
(\varphi, 1)_{T} & =\left(\mathrm{v}_{T}, 1\right)_{T}, & &
\end{aligned}
$$

(or with closure condition $(\varphi, 1)_{T}=\sum_{F \in \mathcal{F}_{T}} \omega_{T F} \vee_{F}$ when $k=0$ and $l=k-1$, see Rem. 2.1). Clearly, $\Phi_{T}$ is well-defined and injective. Moreover, a straightforward extension of ([2], Lem. 3.1) shows that $\underline{\underline{1}}_{T}^{k, l}: V_{T}^{k, l} \rightarrow \underline{\mathrm{U}}_{T}^{k, l}$ is injective. Thus, $\underline{I}_{T}^{k, l}$ is an isomorphism between the space of $\underline{\mathrm{U}}_{T}^{k, l}$ considered in the HHO method and the space of virtual functions $V_{T}^{k, l}$.

Define now the projection $\Pi_{T}^{k+1}: V_{T}^{k, l} \rightarrow \mathbb{P}_{d}^{k+1}(T)$ such that

$$
\Pi_{T}^{k+1} \varphi:=p_{T}^{k+1} \underline{1}_{T}^{k, l} \varphi
$$

Clearly, for any $\varphi \in V_{T}^{k, l}, \Pi_{T}^{k+1} \varphi$ is computable from the function $\underline{\mathrm{I}}_{T}^{k, l} \varphi \in \underline{\mathrm{U}}_{T}^{k, l}$. To reformulate the penalty term, we introduce the map $\delta_{T}^{k, l}: V_{T}^{k, l} \rightarrow \mathrm{U}_{\partial T}^{k}$ such that

$$
\delta_{T}^{k, l} \varphi:=\pi_{\partial T}^{k} \varphi-\pi_{\partial T}^{k} \pi_{T}^{l} \varphi
$$

When $l \leqslant k$, we simply have $\delta_{T}^{k, l} \varphi=\pi_{\partial T}^{k} \varphi-\pi_{T}^{l} \varphi$. For all $\varphi \in V_{T}^{k, l}$, we infer that

$$
\begin{aligned}
\pi_{\partial T}^{k}\left(P_{T}^{k+1, l} \underline{\mathfrak{l}}_{T}^{k, l} \varphi-\left(\underline{\mathbf{l}}_{T}^{k, l} \varphi\right)_{\partial T}\right) & =\pi_{\partial T}^{k}\left(\pi_{T}^{l} \varphi+p_{T}^{k+1} \underline{\mathbf{l}}_{T}^{k, l} \varphi-\pi_{T}^{l} p_{T}^{k+1} \underline{\mathbf{l}}_{T}^{k, l} \varphi-\pi_{\partial T}^{k} \varphi\right) \\
& =\pi_{\partial T}^{k}\left(p_{T}^{k+1} \underline{\mathbf{l}}_{T}^{k, l} \varphi-\varphi-\pi_{T}^{l}\left(p_{T}^{k+1} \underline{\mathfrak{l}}_{T}^{k, l} \varphi-\varphi\right)\right) \\
& =\delta_{T}^{k, l}\left(\Pi_{T}^{k+1} \varphi-\varphi\right),
\end{aligned}
$$

where we have used the definition (2.5) of $P_{T}^{k+1, l}$ in the first line, the fact that $\pi_{\partial T}^{k}\left(\pi_{\partial T}^{k} \varphi\right)=\pi_{\partial T}^{k} \varphi$ in the second line, and the definitions (2.14) and (2.15) of the maps $\Pi_{T}^{k+1}$ and $\delta_{T}^{k, l}$ to conclude. Then, setting $\tau_{\partial T \mid F}=h_{F}^{-1}$ for all $F \in \mathcal{F}_{T}$ and defining the following bilinear forms on $V_{T}^{k, l} \times V_{T}^{k, l}$ :

$$
\begin{aligned}
& \tilde{a}_{T}(\psi, \varphi)=\left(\nabla \Pi_{T}^{k+1} \psi, \nabla \Pi_{T}^{k+1} \varphi\right)_{T}+\widetilde{s}_{T}(\psi, \varphi), \\
& \tilde{s}_{T}(\psi, \varphi)=\left(\tau_{\partial T} \delta_{T}^{k, l}\left(\Pi_{T}^{k+1} \psi-\psi\right), \delta_{T}^{k, l}\left(\Pi_{T}^{k+1} \varphi-\varphi\right)\right)_{\partial T},
\end{aligned}
$$

it is a simple matter to realize that

$$
\tilde{a}_{T}(\psi, \varphi)=a_{T}\left(\underline{I}_{T}^{k, l} \psi, \underline{1}_{T}^{k, l} \varphi\right) \quad \text { and } \quad \tilde{s}_{T}(\psi, \varphi)=s_{T}\left(\underline{\underline{L}}_{T}^{k, l} \psi, \underline{1}_{T}^{k, l} \varphi\right) .
$$

We can observe, at this point, that, when $l=k-1$, the consistent contribution in $\widetilde{a}_{T}$ is analogous to ([2], Eq. (3.17)), whereas the stabilization $\widetilde{s}_{T}$ is a special instance of the one appearing in the right-hand side of ([2], Eq. (3.21)), and can be interpreted as a (computable) least-squares penalty of the difference between a function $\varphi$ in the space $V_{T}^{k, l}$ and its computable projection $\Pi_{T}^{k+1} \varphi$ defined by (2.14). 


\subsection{Static condensation}

We characterize the solution provided by the HHO method by that of a static condensation technique which allows for an efficient implementation of the method. We use a notation inspired from [9].

We start by noting that the equations (2.12) defining the HHO method can be rewritten as

$$
\begin{array}{rlrl}
\forall T \in \mathcal{T}_{h}, & a_{T}\left(\underline{\mathrm{u}}_{T},\left(\mathrm{v}_{T}, 0\right)\right)=\left(f, \mathrm{v}_{T}\right)_{T} & & \forall \mathrm{v}_{T} \in \mathrm{U}_{T}^{l}, \\
a_{h}\left(\underline{\mathrm{u}}_{h},\left(0, \mathrm{v}_{\mathcal{F}_{h}}\right)\right)=0 & & \forall \mathrm{v}_{\mathcal{F}_{h}} \in \mathbf{U}_{\mathcal{F}_{h}, 0}^{k} .
\end{array}
$$

Then, we show that the first set of equations define local problems which allow us to express $\mathbf{u}_{T}$ in terms of $\mathbf{u}_{\partial T}$ and $f_{T}:=f_{\mid T}$ for all cells $T \in \mathcal{T}_{h}$. Finally, we show that the second equation defines a single global problem whose solution is $\mathbf{u}_{\mathcal{F}_{h}}$. We conclude by providing a characterization of the approximate solution in terms of these problems.

Let us first introduce the so-called local problems. Given $\lambda \in \mathrm{U}_{\partial T}^{k}$, define $\mathfrak{U}_{T}^{\lambda} \in \mathrm{U}_{T}^{l}$ as the solution of the local problem

$$
a_{T}\left(\left(\mathfrak{U}_{T}^{\lambda}, 0\right),\left(\mathrm{v}_{T}, 0\right)\right)=-a_{T}\left((0, \lambda),\left(\mathrm{v}_{T}, 0\right)\right) \quad \forall \mathrm{v}_{T} \in \mathrm{U}_{T}^{l},
$$

which, letting $\underline{\mathfrak{U}}_{T}^{\lambda}:=\left(\mathfrak{U}_{T}^{\lambda}, \lambda\right) \in \underline{\cup}_{T}^{k, l}$ and using linearity, rewrites

$$
a_{T}\left(\underline{\mathfrak{U}}_{T}^{\lambda},\left(\mathrm{v}_{T}, 0\right)\right)=0 \quad \forall \mathrm{v}_{T} \in \mathrm{U}_{T}^{l} .
$$

Similarly, given $\phi \in L^{2}(T)$, define $\mathfrak{U}_{T}^{\phi} \in \mathrm{U}_{T}^{l}$ as the solution of the local problem

$$
a_{T}\left(\left(\mathfrak{U}_{T}^{\phi}, 0\right),\left(\mathfrak{v}_{T}, 0\right)\right)=\left(\phi, \mathfrak{v}_{T}\right)_{T} \quad \forall \mathfrak{v}_{T} \in \mathrm{U}_{T}^{l} .
$$

Clearly, by the stability property (2.7), both (2.17) and (2.18) are well-posed since $\|\cdot\|_{a, T}$ is a norm on the zero-trace subspace of $\underline{\mathbf{U}}_{T}^{k, l}$. Moreover, by linearity, we can express in each mesh cell $T \in \mathcal{T}_{h}$ the solution $\underline{\mathbf{u}}_{T}$ in terms of the local datum $f_{T}$ and of the local face-based components $\mathbf{u}_{\partial T}$. Indeed, in view of (2.17) with $\lambda=\mathrm{u}_{\partial T}$ and (2.18) with $\phi=f_{T},(2.16 \mathrm{a})$ yields

$$
\underline{\mathrm{u}}_{T}=\left(\mathfrak{U}_{T}^{\mathrm{u}_{\partial T}}+\mathfrak{U}_{T}^{f_{T}}, \mathrm{u}_{\partial T}\right) \quad \forall T \in \mathcal{T}_{h} .
$$

With obvious notation, we infer that $\underline{\mathrm{u}}_{h}=\left(\mathfrak{U}_{h}^{\mathrm{u}_{\mathcal{F}}}+\mathfrak{U}_{h}^{f}, \mathrm{u}_{\mathcal{F}_{h}}\right)$, which we rewrite in the form

$$
\underline{\mathrm{u}}_{h}=\underline{\mathfrak{U}}_{h}^{\mathrm{u}_{\mathcal{F}_{h}}}+\underline{\mathfrak{U}}_{h}^{f}, \quad \underline{\mathfrak{u}}_{h}^{\mathrm{u}_{\mathcal{F}_{h}}}:=\left(\mathfrak{U}_{h}^{\mathrm{u}_{\mathcal{F}_{h}}}, \mathrm{u}_{\mathcal{F}_{h}}\right), \quad \underline{\mathfrak{U}}_{h}^{f}:=\left(\mathfrak{U}_{h}^{f}, 0\right) .
$$

Now we turn to the global problem defining $\mathbf{u}_{\mathcal{F}_{h}}$. In view of $(2.16 \mathrm{~b})$ and (2.19), we have that, for all $\mathbf{v}_{\mathcal{F}_{h}} \in$ $\mathrm{U}_{\mathcal{F}_{h}, 0}^{k}$, setting $\underline{\mathfrak{U}}_{h}^{\mathrm{v}_{\mathcal{F}_{h}}}:=\left(\mathfrak{U}_{h}^{\mathrm{v}_{\mathcal{F}_{h}}}, \mathrm{v}_{\mathcal{F}_{h}}\right) \in \underline{\mathrm{U}}_{h, 0}^{k, l}$,

$$
\begin{aligned}
& 0=a_{h}\left(\underline{\mathbf{u}}_{h},\left(0, \mathbf{v}_{\mathcal{F}_{h}}\right)\right) \\
& =a_{h}\left(\underline{\mathrm{u}}_{h}, \underline{\mathfrak{U}}_{h}^{\mathrm{v} \mathcal{F}_{h}}\right)-a_{h}\left(\underline{\mathrm{U}}_{h},\left(\mathfrak{U}_{h}^{\mathrm{V}_{\mathcal{F}}}, 0\right)\right) \\
& =a_{h}\left(\underline{\mathrm{u}}_{h}, \underline{\mathfrak{U}}_{h}^{\mathrm{v}_{\mathcal{F}}}\right)-a_{h}\left(\underline{\mathfrak{U}}_{h}^{\mathrm{u} \mathcal{F}_{h}},\left(\mathfrak{U}_{h}^{\mathrm{v}^{\mathcal{F}_{h}}}, 0\right)\right)-a_{h}\left(\underline{\mathfrak{U}}_{h}^{f},\left(\mathfrak{U}_{h}^{\mathrm{v}_{\mathcal{F}}}, 0\right)\right) .
\end{aligned}
$$

But, using the definition (2.11) of $a_{h}$ followed by the local problems (2.17) with $\lambda=\mathrm{u}_{\partial T}$ and $\boldsymbol{v}_{T}=\mathfrak{U}_{T}^{\mathrm{v}_{\partial T}}$ and (2.18) with $\phi=f_{T}$ and $\vee_{T}=\mathfrak{U}_{T}^{v_{\partial T}}$, we infer that

$$
\begin{aligned}
a_{h}\left(\underline{\mathfrak{U}}_{h}^{\mathrm{u}_{\mathcal{F}}},\left(\mathfrak{U}_{h}^{\mathrm{v}_{\mathcal{F}}}, 0\right)\right) & =\sum_{T \in \mathcal{T}_{h}} a_{T}\left(\underline{\mathfrak{U}}_{T}^{\mathrm{u}_{\supset} T},\left(\mathfrak{U}_{T}^{\mathrm{v} T}, 0\right)\right)=0, \\
a_{h}\left(\underline{\mathfrak{U}}_{h}^{f},\left(\mathfrak{U}_{h}^{\mathrm{v}_{\mathcal{F}}}, 0\right)\right) & =\sum_{T \in \mathcal{T}_{h}} a_{T}\left(\left(\mathfrak{U}_{T}^{f_{T}}, 0\right),\left(\mathfrak{U}_{T}^{\mathrm{v} T}, 0\right)\right)=\sum_{T \in \mathcal{T}_{h}}\left(f, \mathfrak{U}_{T}^{\mathrm{v}_{\partial T}}\right)_{T} .
\end{aligned}
$$


Moreover, exploiting the symmetry of the bilinear form $a_{h}$, recalling (2.11), and using the local problem (2.17) with $\lambda=\mathrm{v}_{\partial T}$ and $\mathbf{v}_{T}=\mathfrak{U}_{T}^{f_{T}}$, we infer that

$$
a_{h}\left(\underline{\mathfrak{U}}_{h}^{f}, \underline{\mathfrak{U}}_{h}^{\mathrm{V}_{\mathcal{F}}}\right)=a_{h}\left(\underline{\mathfrak{U}}_{h}^{\mathrm{V}_{\mathcal{F}}}, \underline{\mathfrak{U}}_{h}^{f}\right)=\sum_{T \in \mathcal{T}_{h}} a_{T}\left(\underline{\mathfrak{U}}_{T}^{\mathrm{\vee}_{T} T},\left(\mathfrak{U}_{T}^{f_{T}}, 0\right)\right)=0,
$$

so that, recalling the decomposition $(2.19)$ of $\underline{\mathbf{u}}_{h}$, we conclude that

$$
a_{h}\left(\underline{\mathrm{u}}_{h}, \underline{\mathfrak{U}}_{h}^{\mathrm{v}_{\mathcal{F}}}\right)=a_{h}\left(\underline{\mathfrak{U}}_{h}^{\mathrm{u} \mathcal{F}_{h}}+\underline{\mathfrak{U}}_{h}^{f}, \underline{\mathfrak{U}}_{h}^{\mathrm{v}_{\mathcal{F}}}\right)=a_{h}\left(\underline{\mathfrak{U}}_{h}^{\mathrm{u} \mathcal{F}_{h}}, \underline{\mathfrak{U}}_{h}^{\mathrm{V}_{\mathcal{F}}}\right) .
$$

Plugging (2.21) and (2.22) into the last line of (2.20), the global problem (2.16b) rewrites: Find $\mathbf{u}_{\mathcal{F}_{h}} \in \mathrm{U}_{\mathcal{F}_{h}, 0}^{k}$ such that

$$
a_{h}\left(\left(\mathfrak{U}_{h}^{\mathrm{u}_{\mathcal{F}}}, \mathrm{u}_{\mathcal{F}_{h}}\right),\left(\mathfrak{U}_{h}^{\mathrm{v}_{\mathcal{F}}}, \mathrm{v}_{\mathcal{F}_{h}}\right)\right)=\sum_{T \in \mathcal{T}_{h}}\left(f, \mathfrak{U}_{T}^{\mathrm{v}_{T} T}\right)_{T} \quad \forall \mathrm{v}_{\mathcal{F}_{h}} \in \mathrm{U}_{\mathcal{F}_{h}, 0}^{k}
$$

The well-posedness of this problem follows from the stability of $a_{h}$ together with the well-posedness of the local problems (2.17). We can now summarize our results on the static condensation procedure.

Proposition 2.4 (Characterization of the approximate solution). The solution $\underline{\underline{u}}_{h} \in \underline{\mathrm{U}}_{h}^{k, l}$ given by the HHO method (2.12) can be expressed as (2.19), where $\mathfrak{U}_{h}^{\mathrm{u}_{\mathcal{F}}}$ and $\mathfrak{U}_{h}^{f}$ are defined cellwise as the solutions of the local problems (2.17) and (2.18), and $\mathrm{u}_{\mathcal{F}_{h}} \in \mathrm{U}_{\mathcal{F}_{h}, 0}^{k}$ is the only solution of the problem (2.23).

\section{NumERICAL-TRACE FORMULATION OF HHO METHOD}

In this section, we provide a reinterpretation of the global problem

$$
a_{h}\left(\underline{\mathrm{u}}_{h},\left(0, \mathrm{v}_{\mathcal{F}_{h}}\right)\right)=0 \quad \forall \mathrm{v}_{\mathcal{F}_{h}} \in \mathrm{U}_{\mathcal{F}_{h}, 0}^{k} .
$$

as a transmission condition. In this way, we identify the numerical trace of the flux and show, in a different way from the one proposed in [15], that the method is locally conservative. Finally, we use this information to suitably rewrite the equations defining the local problems, namely,

$$
a_{T}\left(\underline{\mathbf{u}}_{T},\left(\mathrm{v}_{T}, 0\right)\right)=\left(f, \mathrm{v}_{T}\right)_{T} \quad \forall \mathrm{v}_{T} \in \bigcup_{T}^{l}, \quad \forall T \in \mathcal{T}_{h} .
$$

\subsection{The global problem as a transmission condition}

Our goal is to rewrite the bilinear form of the global problem in such a way that the numerical traces can be easily identified. Since $a_{h}$ is assembled cellwise, see (2.11), we can work on a single mesh cell $T \in \mathcal{T}_{h}$. Thus we have, using the definition (2.3) of $a_{T}$ and $(2.2)$ of $p_{T}^{k+1} \underline{\mathrm{v}}_{T}$ with $\underline{\mathrm{v}}_{T}=\left(0, \mathrm{v}_{\partial T}\right)$,

$$
\begin{aligned}
a_{T} & \left.\underline{\mathbf{u}}_{T},\left(0, \mathrm{v}_{\partial T}\right)\right) \\
& =\left(\boldsymbol{\kappa} \nabla p_{T}^{k+1} \underline{\mathbf{u}}_{T}, \boldsymbol{\nabla} p_{T}^{k+1}\left(0, \mathrm{v}_{\partial T}\right)\right)_{T}+\left(\tau_{\partial T} \pi_{\partial T}^{k}\left(P_{T}^{k+1, l} \underline{\mathbf{u}}_{T}-\mathrm{u}_{\partial T}\right), \pi_{\partial T}^{k}\left(P_{T}^{k+1, l}\left(0, \mathrm{v}_{\partial T}\right)-\mathrm{v}_{\partial T}\right)\right)_{\partial T} \\
& =\left(\boldsymbol{\kappa} \nabla p_{T}^{k+1} \underline{\mathbf{u}}_{T} \cdot \boldsymbol{n}_{T}, \mathrm{v}_{\partial T}\right)_{\partial T}+\left(\tau_{\partial T} \pi_{\partial T}^{k}\left(P_{T}^{k+1, l} \underline{\mathbf{u}}_{T}-\mathrm{u}_{\partial T}\right), \pi_{\partial T}^{k}\left(P_{T}^{k+1, l}\left(0, \mathrm{v}_{\partial T}\right)-\mathrm{v}_{\partial T}\right)\right)_{\partial T} .
\end{aligned}
$$

Next, we note that, by definition of $P_{T}^{k+1, l}$, we have that

$$
\begin{aligned}
P_{T}^{k+1, l} \underline{\mathbf{u}}_{T}-\mathbf{u}_{\partial T}= & \mathbf{u}_{T}+p_{T}^{k+1} \underline{\mathbf{u}}_{T}-\pi_{T}^{l} p_{T}^{k+1} \underline{\mathbf{u}}_{T}-\mathbf{u}_{\partial T} \\
= & \mathbf{u}_{T}+p_{T}^{k+1}\left(\mathbf{u}_{T}, \mathbf{u}_{\partial T}\right)-\pi_{T}^{l} p_{T}^{k+1}\left(\mathbf{u}_{T}, \mathbf{u}_{\partial T}\right)-\mathbf{u}_{\partial T} \\
= & \mathbf{u}_{T}+p_{T}^{k+1}\left(\mathbf{u}_{T}, \mathbf{u}_{T}\right)+p_{T}^{k+1}\left(0, \mathbf{u}_{\partial T}-\mathbf{u}_{T}\right) \\
& -\pi_{T}^{l} p_{T}^{k+1}\left(\mathbf{u}_{T}, \mathbf{u}_{T}\right)-\pi_{T}^{l} p_{T}^{k+1}\left(0, \mathbf{u}_{\partial T}-\mathbf{u}_{T}\right)-\mathbf{u}_{\partial T} \\
= & \mathbf{u}_{T}+p_{T}^{k+1}\left(0, \mathbf{u}_{\partial T}-\mathbf{u}_{T}\right)-\pi_{T}^{l} p_{T}^{k+1}\left(0, \mathbf{u}_{\partial T}-\mathbf{u}_{T}\right)-\mathbf{u}_{\partial T} \\
= & \mathbf{u}_{T}-\mathbf{u}_{\partial T}+p_{T}^{k+1}\left(0, \mathbf{u}_{\partial T}-\mathbf{u}_{T}\right)-\pi_{T}^{l} p_{T}^{k+1}\left(0, \mathbf{u}_{\partial T}-\mathbf{u}_{T}\right),
\end{aligned}
$$


where, to pass to the fourth line, we have used the fact that $p_{T}^{k+1}\left(\mathbf{u}_{T}, \mathbf{u}_{T}\right)=\pi_{T}^{l} p_{T}^{k+1}\left(\mathbf{u}_{T}, \mathbf{u}_{T}\right)(c f .(2.2)$ and Rem. 2.1 for the case $k=0$ and $l=k-1)$. Then for any $\lambda \in \mathrm{U}_{\partial T}^{k}$, we define $r_{\partial T}^{k}(\lambda)$ as the element of $\mathrm{U}_{\partial T}^{k}$ such that

$$
r_{\partial T}^{k}(\lambda):=\pi_{\partial T}^{k}\left(\lambda-p_{T}^{k+1}(0, \lambda)+\pi_{T}^{l} p_{T}^{k+1}(0, \lambda)\right)
$$

In this way, accounting for (3.2), we can write

$$
\pi_{\partial T}^{k}\left(P_{T}^{k+1, l} \underline{\mathbf{u}}_{T}-\mathbf{u}_{\partial T}\right)=r_{\partial T}^{k}\left(\mathbf{u}_{T}-\mathbf{u}_{\partial T}\right),
$$

and so, plugging this expression into (3.1), we obtain

$$
a_{T}\left(\underline{\mathrm{u}}_{T},\left(0, \mathrm{v}_{\partial T}\right)\right)=\left(\boldsymbol{\kappa} \nabla p_{T}^{k+1} \underline{\mathbf{u}}_{T} \cdot \boldsymbol{n}_{T}, \mathrm{v}_{\partial T}\right)_{\partial T}-\left(\tau_{\partial T} r_{\partial T}^{k}\left(\mathrm{u}_{T}-\mathrm{u}_{\partial T}\right), r_{\partial T}^{k}\left(\mathrm{v}_{\partial T}\right)\right)_{\partial T} .
$$

Defining the adjoint $r_{\partial T}^{k *}$ of $r_{\partial T}^{k}$, as the unique element of $\mathrm{U}_{\partial T}^{k}$ such that

$$
\forall \lambda \in \mathrm{U}_{\partial T}^{k}, \quad\left(r_{\partial T}^{k *}(\lambda), \mu\right)_{\partial T}=\left(\lambda, r_{\partial T}^{k}(\mu)\right)_{\partial T} \quad \forall \mu \in \mathrm{U}_{\partial T}^{k},
$$

we finally obtain from (3.4),

$$
a_{T}\left(\underline{\mathbf{u}}_{T},\left(0, \mathrm{v}_{\partial T}\right)\right)=\left(\boldsymbol{\kappa} \nabla p_{T}^{k+1} \underline{\mathbf{u}}_{T} \cdot \boldsymbol{n}_{T}-r_{\partial T}^{k *}\left(\tau_{\partial T} r_{\partial T}^{k}\left(\mathbf{u}_{T}-\mathrm{u}_{\partial T}\right)\right), \mathrm{v}_{\partial T}\right)_{\partial T},
$$

so that the global problem can be expressed as follows:

$$
\sum_{T \in \mathcal{T}_{h}}\left(\widehat{\boldsymbol{q}}_{\underline{\mathbf{u}}_{T}} \cdot \boldsymbol{n}_{T}, \mathbf{v}_{\mathcal{F}_{h}}\right)_{\partial T}=0 \quad \forall \mathrm{v}_{\mathcal{F}_{h}} \in \mathrm{U}_{\mathcal{F}_{h}, 0}^{k},
$$

where

$$
\widehat{\boldsymbol{q}}_{\underline{\underline{u}}_{T}} \cdot \boldsymbol{n}_{T}:=-\boldsymbol{\kappa} \nabla p_{T}^{k+1} \underline{\mathbf{u}}_{T} \cdot \boldsymbol{n}_{T}+r_{\partial T}^{k *}\left(\tau_{\partial T} r_{\partial T}^{k}\left(\mathrm{u}_{T}-\mathrm{u}_{\partial T}\right)\right)
$$

is nothing but the numerical approximation of the normal trace of the exact flux $\boldsymbol{q}:=-\boldsymbol{\kappa} \boldsymbol{\nabla} u$ we sought. The global problem can thus be interpreted as a discrete version of a transmission condition since it enforces the single-valuedness of the normal component of the numerical trace of the flux.

\subsection{Rewriting the equations defining the local problems}

We can now rewrite the equation (2.16a) by using the numerical trace just uncovered. Indeed, for all $T \in \mathcal{T}_{h}$, using the definition (2.3) of $a_{T}$, accounting for (3.3) and (3.5), and concluding with the definition (2.2) of $p_{T}^{k+1}$ with $\underline{\mathrm{v}}_{T}=\left(\mathrm{v}_{T}, 0\right)$ and $w=p_{T}^{k+1} \underline{\mathrm{u}}_{T}$, we have

$$
\begin{aligned}
a_{T}\left(\underline{\mathbf{u}}_{T},\left(\mathbf{v}_{T}, 0\right)\right) & =\left(\boldsymbol{\kappa} \nabla p_{T}^{k+1} \underline{\mathbf{u}}_{T}, \boldsymbol{\nabla} p_{T}^{k+1}\left(\mathbf{v}_{T}, 0\right)\right)_{T}+\left(\tau_{\partial T} \pi_{\partial T}^{k}\left(P_{T}^{k+1, l} \underline{\mathbf{u}}_{T}-\mathbf{u}_{\partial T}\right), \pi_{\partial T}^{k}\left(P_{T}^{k+1, l}\left(\mathbf{v}_{T}, 0\right)\right)\right)_{\partial T} \\
& =\left(\boldsymbol{\kappa} \nabla p_{T}^{k+1} \underline{\mathbf{u}}_{T}, \boldsymbol{\nabla} p_{T}^{k+1}\left(\mathrm{v}_{T}, 0\right)\right)_{T}+\left(r_{\partial T}^{k *}\left(\tau_{\partial T} r_{\partial T}^{k}\left(\mathbf{u}_{T}-\mathbf{u}_{\partial T}\right)\right), \mathbf{v}_{T}\right)_{\partial T} \\
& =\left(\boldsymbol{\kappa} \nabla p_{T}^{k+1} \underline{\mathbf{u}}_{T}, \nabla \mathbf{v}_{T}\right)_{T}+\left(-\boldsymbol{\kappa} \nabla p_{T}^{k+1} \underline{\mathbf{u}}_{T} \cdot \boldsymbol{n}_{T}+r_{\partial T}^{k *}\left(\tau_{\partial T} r_{\partial T}^{k}\left(\mathbf{u}_{T}-\mathbf{u}_{\partial T}\right)\right), \mathbf{v}_{T}\right)_{\partial T} .
\end{aligned}
$$

Thus, (2.16a) is equivalent to

$$
\forall T \in \mathcal{T}_{h}, \quad\left(\boldsymbol{\kappa} \nabla p_{T}^{k+1} \underline{\mathbf{u}}_{T}, \boldsymbol{\nabla} \mathbf{v}_{T}\right)_{T}+\left(\widehat{\boldsymbol{q}}_{\underline{\mathbf{u}}_{T}} \cdot \boldsymbol{n}_{T}, \mathbf{v}_{T}\right)_{\partial T}=\left(f, \mathbf{v}_{T}\right)_{T} \quad \forall \mathbf{v}_{T} \in \mathrm{U}_{T}^{l},
$$

which, since the numerical trace of the flux is single-valued, expresses a local conservation condition. We have thus proven that the HHO method has the following equivalent formulation.

Proposition 3.1 (Numerical-trace formulation). The solution $\underline{\mathrm{u}}_{h} \in \underline{\mathrm{U}}_{h, 0}^{k, l}$ provided by the HHO method (2.12) satisfies the following local problems: for all $T \in \mathcal{T}_{h}$,

$$
\begin{array}{rlrl}
\left(\boldsymbol{\kappa} \nabla p_{T}^{k+1} \underline{\underline{u}}_{T}, \boldsymbol{\nabla} w\right)_{T}+\left(\mathbf{u}_{T}, \boldsymbol{\nabla} \cdot(\boldsymbol{\kappa} \boldsymbol{\nabla} w)\right)_{T} & =\left(\mathbf{u}_{\partial T}, \boldsymbol{\kappa} \boldsymbol{\nabla} w \cdot \boldsymbol{n}_{T}\right)_{\partial T} & & \forall w \in \mathbb{P}_{d}^{k+1}(T), \\
\left(\boldsymbol{\kappa} \boldsymbol{\nabla} p_{T}^{k+1} \underline{\mathbf{u}}_{T}, \boldsymbol{\nabla} \mathbf{v}_{T}\right)_{T}+\left(\widehat{\boldsymbol{q}}_{\underline{\underline{u}}_{T}} \cdot \boldsymbol{n}_{T}, \mathbf{v}_{T}\right)_{\partial T}=\left(f, \mathbf{v}_{T}\right)_{T} & & \forall \mathbf{v}_{T} \in \mathbf{U}_{T}^{l},
\end{array}
$$


where the numerical trace of the flux is given by

$$
\widehat{\boldsymbol{q}}_{\underline{\mathbf{u}}_{T}} \cdot \boldsymbol{n}_{T}:=-\boldsymbol{\kappa} \nabla p_{T}^{k+1} \underline{\mathbf{u}}_{T} \cdot \boldsymbol{n}_{T}+r_{\partial T}^{k *}\left(\tau_{\partial T} r_{\partial T}^{k}\left(\mathrm{u}_{T}-\mathrm{u}_{\partial T}\right)\right)
$$

and satisfies the transmission condition

$$
\sum_{T \in \mathcal{T}_{h}}\left(\widehat{\boldsymbol{q}}_{\underline{\mathbf{u}}_{T}} \cdot \boldsymbol{n}_{T}, \mathrm{v}_{\mathcal{F}_{h}}\right)_{\partial T}=0 \quad \forall \mathrm{v}_{\mathcal{F}_{h}} \in \mathrm{U}_{\mathcal{F}_{h}, 0}^{k}
$$

\section{Comparison With HDG Methods}

In this section, we show that the numerical-trace formulation of the HHO methods fits in the framework of HDG methods introduced in [9]. This allows us to compare the HHO method with other HDG methods. It also allows us to incorporate into the family of HDG methods the subtle way of defining the HHO numerical trace for the flux, giving thus rise to new HDG methods.

\subsection{The HDG framework}

We begin by recalling the general framework defining the HDG methods. We define the spaces

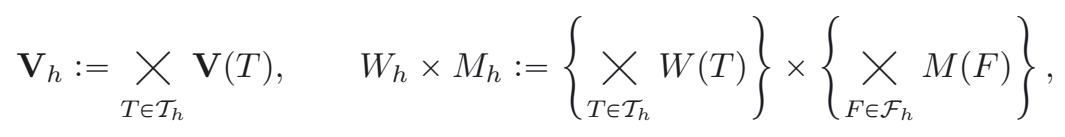

and $M_{h, 0}:=\left\{\widehat{w} \in M_{h}: \quad \widehat{w}=0\right.$ on $\left.\partial \Omega\right\}$. The HDG discretization of problem (1.1) consists in seeking $\left(\mathbf{q}_{h}, u_{h}, \widehat{u}_{h}\right) \in \mathbf{V}_{h} \times W_{h} \times M_{h, 0}$ as the solution of the local problems

$$
\begin{array}{rlrl}
\left(\boldsymbol{\kappa}^{-1} \mathbf{q}_{h}, \mathbf{v}\right)_{T}-\left(u_{h}, \boldsymbol{\nabla} \cdot \mathbf{v}\right)_{T}+\left(\widehat{u}_{h}, \mathbf{v} \cdot \boldsymbol{n}_{T}\right)_{\partial T} & =0 & & \forall \mathbf{v} \in \mathbf{V}(T), \\
-\left(\mathbf{q}_{h}, \boldsymbol{\nabla} w\right)_{T}+\left(\widehat{\mathbf{q}}_{h} \cdot \boldsymbol{n}_{T}, w\right)_{\partial T} & =(f, w)_{T} & & \forall w \in W(T), \\
\widehat{\mathbf{q}}_{h} \cdot \boldsymbol{n}_{T}:=\mathbf{q}_{h} \cdot \boldsymbol{n}_{T}+\alpha\left(u_{h}-\widehat{u}_{h}\right) & \text { on } \partial T, \\
\sum_{T \in \mathcal{T}_{h}}\left(\widehat{\mathbf{q}}_{h} \cdot \boldsymbol{n}_{T}, \widehat{w}\right)_{\partial T} & =0 & & \forall \widehat{w} \in M_{h, 0},
\end{array}
$$

where the last equation is the transmission condition. To complete this framework, a new approximation of the potential, $u_{h}^{*}$, is defined in a suitable manner; see the examples in $[10,11]$.

The above formulation is usually considered to be a mixed formulation. A small variation, which has been called the extended form of the mixed formulation, see $[1,8,26]$, can be trivially used to define HDG methods. Roughly speaking, it avoids using the tensor $\boldsymbol{\kappa}^{-1}$ and only uses the tensor $\boldsymbol{\kappa}$ by discretizing the equation $\mathbf{q}=-\boldsymbol{\kappa} \nabla u$ instead of the equation $\boldsymbol{\kappa}^{-1} \mathbf{q}=-\nabla u$. In linear elasticity, $\boldsymbol{\kappa}$ corresponds to the standard constitutive tensor whereas $\boldsymbol{\kappa}^{-1}$ to the so-called compliance tensor. To work with the constitutive tensor is usually preferred in the case of nonlinear elasticity. This formulation has been used, for example, for the HDG method for linear and nonlinear elasticity in $[25,34,35]$.

The local spaces $\mathbf{V}(T), W(T), M(F)$ and the stabilization function $\alpha$, as well as the post-processing $u_{h}^{*}$, determine the different HDG methods. In particular, the HHO methods of Section 2.2 are obtained for the choice

$$
\boldsymbol{V}(T):=\boldsymbol{\kappa} \nabla \mathbb{P}_{d}^{k+1}(T), \quad W(T):=\mathbb{P}_{d}^{l}(T), \quad M(F):=\mathbb{P}_{d-1}^{k}(F) \quad \text { and } \quad \alpha:=r_{\partial T}^{k, *}\left(\tau_{\partial T} r_{\partial T}^{k}\right),
$$

and the postprocessing $u_{h}^{*}:=p_{h}^{k+1}\left(u_{h}, \widehat{u}_{h}\right)$, in the HDG notation. 
TABLE 1. Comparison of various methods fitting in the HDG framework (4.2) with $M(F):=$ $\mathbb{P}_{d-1}^{k}(F)$. The methods are defined by the local spaces $\mathbf{V}(T), W(T)(c f .(4.1))$, and $u_{h}^{*}$ which determines the numerical trace of the flux which is of the form (4.3). The notation $\operatorname{HHO}(l)$ corresponds to the value of the integer $l$ in (2.1). The corresponding orders of convergence are provided for the $L^{2}(\Omega)$-norm of the error in the approximate flux $\boldsymbol{q}_{h}$ and in the approximate potential $u_{h}^{*}$.

\begin{tabular}{|c|c|c|c|c|c|c|c|}
\hline Ref. & & $\mathbf{V}(T)$ & $W(T)$ & $u_{h}^{*}$ & $\tau_{\partial T \mid F}$ & flux & potential \\
\hline \multirow{2}{*}{ LDG-H } & \multirow{2}{*}[7,9]{} & \multirow{2}{*}{$\mathbb{P}_{d}^{k}(T)^{d}$} & \multirow{2}{*}{$\mathbb{P}_{d}^{k}(T)$} & \multirow{2}{*}{$u_{h}$} & $\left\{\kappa_{T F} / h\right.$ & $k$ & $k+1$ \\
\hline & & & & & $\kappa_{T F}$ & $k+1 / 2$ & $k+1$ \\
\hline (A) & {$[29,31]$} & $\mathbb{P}_{d}^{k}(T)^{d}$ & $\mathbb{P}_{d}^{k+1}(T)$ & $u_{h}$ & $\kappa_{T F} / h$ & $k+1$ & $k+2$ \\
\hline (B) & new & $\mathbb{P}_{d}^{k}(T)^{d}$ & $\mathbb{P}_{d}^{k}(T)$ & $\mathfrak{p}_{h}^{k+1}\left(u_{h}, \widehat{u}_{h}\right)$ & $\kappa_{T F} / h$ & $k+1$ & $k+2$ \\
\hline (C) & new & $\mathbb{P}_{d}^{k}(T)^{d}$ & $\mathbb{P}_{d}^{k-1}(T)$ & $\mathfrak{p}_{h}^{k+1}\left(u_{h}, \widehat{u}_{h}\right)$ & $\kappa_{T F} / h$ & $k+1$ & $k+2$ \\
\hline $\operatorname{HHO}(k+1)$ & new & $\kappa \boldsymbol{\nabla} \mathbb{P}_{d}^{k+1}(T)$ & $\mathbb{P}_{d}^{k+1}(T)$ & $p_{h}^{k+1}\left(u_{h}, \widehat{u}_{h}\right)$ & $\kappa_{T F} / h$ & $k+1$ & $k+2$ \\
\hline $\operatorname{HHO}(k)$ & {$[16,18]$} & $\kappa \boldsymbol{\nabla} \mathbb{P}_{d}^{k+1}(T)$ & $\mathbb{P}_{d}^{k}(T)$ & $p_{h}^{k+1}\left(u_{h}, \widehat{u}_{h}\right)$ & $\kappa_{T F} / h$ & $k+1$ & $k+2$ \\
\hline $\operatorname{HHO}(k-1)$ & {$[30]$} & $\kappa \nabla \mathbb{P}_{d}^{k+1}(T)$ & $\mathbb{P}_{d}^{k-1}(T)$ & $p_{h}^{k+1}\left(u_{h}, \widehat{u}_{h}\right)$ & $\kappa_{T F} / h$ & $k+1$ & $k+2$ \\
\hline
\end{tabular}

\subsection{Comparison with other HDG methods}

We compare several HDG methods with the HHO method in Table 1, where we display the local spaces, the numerical trace of the flux and the theoretical orders of convergence of error in the flux, $\left\|\boldsymbol{q}-\boldsymbol{q}_{h}\right\|$, and in the potential, $\left\|u-u_{h}^{*}\right\|$. The orders of convergence for the methods (B), (C), and $\mathrm{HHO}(l)$ with $l \in\{k-1, k+1\}$ can be established by (minor) adaptations of the proofs for the $\operatorname{HHO}(k)$ variant; $c f .[17,18]$. Details are omitted for the sake of conciseness. In all the cases considered the numerical flux is of the form

$$
\begin{aligned}
\widehat{\boldsymbol{q}}_{h} \cdot \boldsymbol{n} & :=\mathbf{q}_{h} \cdot \boldsymbol{n}+r_{\partial T}^{k *}\left(\tau_{\partial T} r_{\partial T}^{k}\left(u_{h}-\widehat{u}_{h}\right)\right), \\
r_{\partial T}^{k}\left(u_{h}-\widehat{u}_{h}\right) & :=\pi_{\partial T}^{k}\left(u_{h}+u_{h}^{*}-\pi_{W(T)} u_{h}^{*}-\widehat{u}_{h}\right),
\end{aligned}
$$

where $\pi_{W(T)}$ denotes is the $L^{2}$-orthogonal projection onto $W(T)$.

For the methods (B) and (C), the post-processing $u_{h}^{*}$ is given by the function $\mathfrak{p}_{h}^{k+1}\left(u_{h}, \widehat{u}_{h}\right) \in \mathbb{P}_{d}^{k+1}(T)$ defined as the solution of

$$
\begin{aligned}
\left(\boldsymbol{\nabla} \mathfrak{p}_{h}^{k+1}\left(u_{h}, \widehat{u}_{h}\right), \boldsymbol{\nabla} z\right)_{T} & =-\left(u_{h}, \Delta z\right)_{T}+\left(\widehat{u}_{h}, \boldsymbol{\nabla} z \cdot \boldsymbol{n}\right)_{\partial T} & & \forall z \in \mathbb{P}_{d}^{k+1}(T)^{\perp}, \\
\left(\mathfrak{p}_{h}^{k+1}\left(u_{h}, \widehat{u}_{h}\right), w\right)_{T} & =\left(u_{h}, w\right)_{T} & & \forall w \in W(T) .
\end{aligned}
$$

where $\mathbb{P}_{d}^{k+1}(T)^{\perp}:=\left\{z \in \mathbb{P}_{d}^{k+1}(T) \mid(z, w)_{T}=0 \forall w \in W(T)\right\}$. Note that the operator $\mathfrak{p}_{h}^{k+1}$ is a small variation of the post-processings used in [10,11], where a proper subspace of $W(T)$ is used instead of $W(T)$. Note also that, for the method (A), we have that $W(T)=\mathbb{P}_{d}^{k+1}(T)$ so that $\mathbb{P}_{d}^{k+1}(T)^{\perp}=\{0\}$ and, therefore, $u_{h}^{*}:=\mathfrak{p}_{h}^{k+1}\left(u_{h}, \widehat{u}_{h}\right)=u_{h}$.

For the methods (A), (B) and (C), we have $\pi_{W(T)} u_{h}^{*}=\pi_{W(T)} \mathfrak{p}_{h}^{k+1}\left(u_{h}, \widehat{u}_{h}\right)=u_{h}$, and so

$$
r_{\partial T}^{k}\left(u_{h}-\widehat{u}_{h}\right)=\pi_{\partial T}^{k}\left(u_{h}^{*}-\widehat{u}_{h}\right)=\pi_{\partial T}^{k} u_{h}^{*}-\widehat{u}_{h} .
$$

For the methods $(\mathrm{A})$ and $\operatorname{HHO}(k+1)$, we have, on the other hand, that $u_{h}^{*} \in W(T)=\mathbb{P}_{d}^{k+1}(T)$, and so the penalty term in (4.3) takes a simpler expression ( $c f$. also Rem. 2.2):

$$
r_{\partial T}^{k}\left(u_{h}-\widehat{u}_{h}\right)=\pi_{\partial T}^{k}\left(u_{h}-\widehat{u}_{h}\right)=\pi_{\partial T}^{k} u_{h}-\widehat{u}_{h} .
$$


TABLE 2. Size of the local problem to solve to locally eliminate the flux variable for the HDG (rows 1-4) and HHO (rows 5-7) methods listed in Table 1.

\begin{tabular}{cccc}
\hline $\mathrm{k}$ & $d=1$ & $d=2$ & $d=3$ \\
\hline 0 & 1 & 2 & 3 \\
1 & 2 & 6 & 12 \\
2 & 3 & 12 & 30 \\
3 & 4 & 20 & 60 \\
\hline
\end{tabular}

(A) HDG methods

\begin{tabular}{cccc}
\hline $\mathrm{k}$ & $d=1$ & $d=2$ & $d=3$ \\
\hline 0 & 1 & 2 & 3 \\
1 & 2 & 5 & 9 \\
2 & 3 & 9 & 19 \\
3 & 4 & 14 & 34 \\
\hline
\end{tabular}

(B) HHO methods

As defined in [9], the LDG-H methods are the HDG methods obtained when the local discontinuous Galerkin (LDG) method is used to define the local problems. It is actually the particular case of discontinuous Galerkin methods proposed in [7] for which we have, at each interior face $F \in \mathcal{F}_{h}^{\mathrm{i}}$ such that $F=\partial T^{+} \cap \partial T^{-}$, letting $\tau_{F}^{ \pm}:=\tau_{\partial T^{ \pm} \mid F}$,

$$
\begin{aligned}
& \widehat{u}_{h}=\left(\frac{\tau_{F}^{+}}{\tau_{F}^{-}+\tau_{F}^{+}}\right) u_{h}^{+}+\left(\frac{\tau_{F}^{-}}{\tau_{F}^{-}+\tau_{F}^{+}}\right) u_{h}^{-}+\left(\frac{1}{\tau_{F}^{+}+\tau_{F}^{-}}\right)\left(\boldsymbol{q}_{h}^{+} \cdot \boldsymbol{n}^{+}+\boldsymbol{q}_{h}^{-} \cdot \boldsymbol{n}^{-}\right), \\
& \widehat{\boldsymbol{q}}_{h}=\left(\frac{\tau_{F}^{-}}{\tau_{F}^{-}+\tau_{F}^{+}}\right) \boldsymbol{q}_{h}^{+}+\left(\frac{\tau_{F}^{+}}{\tau_{F}^{-}+\tau_{F}^{+}}\right) \boldsymbol{q}_{h}^{-}+\left(\frac{\tau_{F}^{+} \tau_{F}^{-}}{\tau_{F}^{-}+\tau_{F}^{+}}\right)\left(u_{h}^{+} \boldsymbol{n}^{+}+u_{h}^{-} \boldsymbol{n}^{-}\right) .
\end{aligned}
$$

Here the superscript \pm indicates the traces from both sides of the face. For this choice of numerical traces, we have that

$$
\widehat{\boldsymbol{q}}_{h} \cdot \boldsymbol{n}^{ \pm}=\boldsymbol{q}_{h}^{ \pm} \cdot \boldsymbol{n}+\tau_{F}^{ \pm}\left(u_{h}^{ \pm}-\widehat{u}_{h}\right) .
$$

The orders of convergence of this method were obtained in [7] for general, shape-regular polygonal meshes. The suboptimal order of $k+1 / 2$ is obtained for the approximate flux for $\tau_{F}$ of order one.

The method (A), whose stabilization function we could call the Lehrenfeld/Schöberl stabilization, was suggested in ([29], Rem. 1.2.4) and was recently analyzed in [31]. Extensions to convection-diffusion and linear elasticity can be found in [32,33], respectively. This method uses polynomials of one higher degree for $u_{h}$, and achieves optimal order for the approximate flux, $k+1$, and for the potential, $k+2$, by stabilizing using only the lowest-order part of the difference between cell and face unknowns. The methods (B) and (C) can be considered as novel HHO-inspired variations of this method.

The $\mathrm{HHO}(k)$ method uses the same space for $u_{h}$ as the LDG-H method, but is built upon a reconstruction $u_{h}^{*}$ which uses polynomials of one higher degree. The method achieves optimal orders of convergence for the approximate flux and potential with significantly smaller spaces for the fluxes, namely, $\kappa \nabla \mathbb{P}_{d}^{k+1}(T)$ instead of $\mathbb{P}_{d}^{k}(T)^{d}$. The order of the matrix we need to invert to eliminate the flux variable (when solving the local problems) is only $\left(\begin{array}{c}k+1+d \\ d\end{array}\right)-1$ instead of $d\left(\begin{array}{c}k+d \\ d\end{array}\right), c f$. Table 2. Similar considerations apply for the methods $\operatorname{HHO}(k \pm 1)$.

\subsection{Numerical experiments}

Here, we compare the original $\mathrm{HHO}(k)$ method of $(2.12)$ with the novel $\mathrm{HHO}(k+1)$ variant, $c f$. Table 1 . As pointed out in Remark 2.2, the stabilizing bilinear form takes a very simple expression for $\operatorname{HHO}(k+1), c f .(2.10)$, although this comes at the price of slightly increasing the cost of both the potential reconstruction (2.2) (the number of right-hand sides increases) and of the local problems to be solved for static condensation. For $\Omega=(0,1)^{2}$, we consider the Dirichlet problem corresponding to the exact solution $u=\sin \left(\pi x_{1}\right) \sin \left(\pi x_{2}\right)$ for two values of the diffusion tensors

$$
\boldsymbol{\kappa}_{1}=\boldsymbol{I}_{d}, \quad \boldsymbol{\kappa}_{2}=\left(\begin{array}{cc}
\left(x_{2}-\bar{x}_{2}\right)^{2}+\epsilon\left(x_{1}-\bar{x}_{1}\right)^{2} & -(1-\epsilon)\left(x_{1}-\bar{x}_{1}\right)\left(x_{2}-\bar{x}_{2}\right) \\
-(1-\epsilon)\left(x_{1}-\bar{x}_{1}\right)\left(x_{2}-\bar{x}_{2}\right) & \left(x_{1}-\bar{x}_{1}\right)^{2}+\epsilon\left(x_{2}-\bar{x}_{2}\right)^{2}
\end{array}\right),
$$



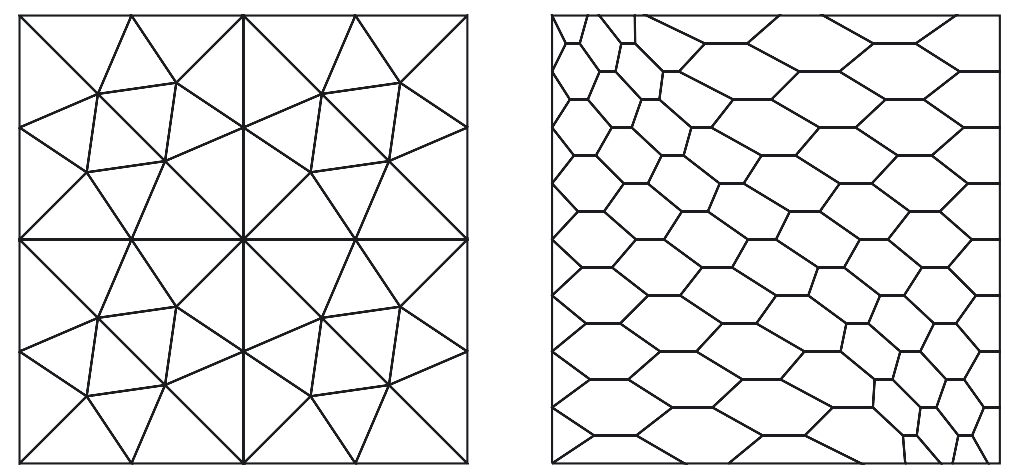

Figure 1. Triangular and (predominantly) hexagonal meshes.

TABLE 3. Triangular mesh family (isotropic homogeneous test case $\boldsymbol{\kappa}=\boldsymbol{\kappa}_{1}$ ).

\begin{tabular}{|c|c|c|c|c|c|c|c|c|}
\hline \multirow[b]{2}{*}{$h$} & \multicolumn{4}{|c|}{$\mathrm{HHO}(k)$} & \multicolumn{4}{|c|}{$\mathrm{HHO}(k+1)$} \\
\hline & $H^{1}$ & ECR & $L^{2}$ & ECR & $H^{1}$ & ECR & $L^{2}$ & ECR \\
\hline \multicolumn{9}{|c|}{$k=0$} \\
\hline $3.07 \times 10^{-2}$ & 0.14 & - & 0.11 & - & 0.14 & - & 0.15 & - \\
\hline $1.54 \times 10^{-2}$ & $7.06 \times 10^{-2}$ & 1.00 & $2.84 \times 10^{-2}$ & 2.01 & $7.04 \times 10^{-2}$ & 0.99 & $3.67 \times 10^{-2}$ & 2.00 \\
\hline $7.68 \times 10^{-3}$ & $3.53 \times 10^{-2}$ & 1.00 & $7.10 \times 10^{-3}$ & 1.99 & $3.53 \times 10^{-2}$ & 0.99 & $9.19 \times 10^{-3}$ & 1.99 \\
\hline $3.84 \times 10^{-3}$ & $1.77 \times 10^{-2}$ & 1.00 & $1.78 \times 10^{-3}$ & 2.00 & $1.77 \times 10^{-2}$ & 1.00 & $2.30 \times 10^{-3}$ & 2.00 \\
\hline $1.92 \times 10^{-3}$ & $8.83 \times 10^{-3}$ & 1.00 & $4.44 \times 10^{-4}$ & 2.00 & $8.83 \times 10^{-3}$ & 1.00 & $5.75 \times 10^{-4}$ & 2.00 \\
\hline \multicolumn{9}{|c|}{$k=1$} \\
\hline $3.07 \times 10^{-2}$ & $1.36 \times 10^{-2}$ & - & $1.16 \times 10^{-2}$ & - & $1.40 \times 10^{-2}$ & - & $1.21 \times 10^{-2}$ & - \\
\hline $1.54 \times 10^{-2}$ & $3.28 \times 10^{-3}$ & 2.06 & $1.46 \times 10^{-3}$ & 3.00 & $3.40 \times 10^{-3}$ & 2.05 & $1.52 \times 10^{-3}$ & 3.01 \\
\hline $7.68 \times 10^{-3}$ & $8.10 \times 10^{-4}$ & 2.01 & $1.83 \times 10^{-4}$ & 2.98 & $8.40 \times 10^{-4}$ & 2.01 & $1.90 \times 10^{-4}$ & 2.99 \\
\hline $3.84 \times 10^{-3}$ & $2.02 \times 10^{-4}$ & 2.00 & $2.28 \times 10^{-5}$ & 3.00 & $2.09 \times 10^{-4}$ & 2.01 & $2.37 \times 10^{-5}$ & 3.00 \\
\hline $1.92 \times 10^{-3}$ & $5.04 \times 10^{-5}$ & 2.00 & $2.86 \times 10^{-6}$ & 2.99 & $5.23 \times 10^{-5}$ & 2.00 & $2.97 \times 10^{-6}$ & 3.00 \\
\hline \multicolumn{9}{|c|}{$k=2$} \\
\hline $3.07 \times 10^{-2}$ & $1.01 \times 10^{-3}$ & - & $9.53 \times 10^{-4}$ & - & $1.04 \times 10^{-3}$ & - & $1.02 \times 10^{-3}$ & - \\
\hline $1.54 \times 10^{-2}$ & $1.22 \times 10^{-4}$ & 3.06 & $6.03 \times 10^{-5}$ & 4.00 & $1.27 \times 10^{-4}$ & 3.05 & $6.48 \times 10^{-5}$ & 4.00 \\
\hline $7.68 \times 10^{-3}$ & $1.50 \times 10^{-5}$ & 3.01 & $3.78 \times 10^{-6}$ & 3.98 & $1.57 \times 10^{-5}$ & 3.00 & $4.06 \times 10^{-6}$ & 3.98 \\
\hline $3.84 \times 10^{-3}$ & $1.87 \times 10^{-6}$ & 3.00 & $2.37 \times 10^{-7}$ & 4.00 & $1.95 \times 10^{-6}$ & 3.01 & $2.54 \times 10^{-7}$ & 4.00 \\
\hline $1.92 \times 10^{-3}$ & $2.33 \times 10^{-7}$ & 3.00 & $1.48 \times 10^{-8}$ & 4.00 & $2.42 \times 10^{-7}$ & 3.01 & $1.59 \times 10^{-8}$ & 4.00 \\
\hline \multicolumn{9}{|c|}{$k=3$} \\
\hline $3.07 \times 10^{-2}$ & $8.49 \times 10^{-5}$ & - & $5.91 \times 10^{-5}$ & - & $8.95 \times 10^{-5}$ & - & $6.49 \times 10^{-5}$ & - \\
\hline $1.54 \times 10^{-2}$ & $5.39 \times 10^{-6}$ & 4.00 & $1.87 \times 10^{-6}$ & 5.01 & $5.70 \times 10^{-6}$ & 3.99 & $2.05 \times 10^{-6}$ & 5.01 \\
\hline $7.68 \times 10^{-3}$ & $3.38 \times 10^{-7}$ & 3.98 & $5.85 \times 10^{-8}$ & 4.98 & $3.58 \times 10^{-7}$ & 3.98 & $6.41 \times 10^{-8}$ & 4.98 \\
\hline $3.84 \times 10^{-3}$ & $2.11 \times 10^{-8}$ & 4.00 & $1.83 \times 10^{-9}$ & 5.00 & $2.24 \times 10^{-8}$ & 4.00 & $2.01 \times 10^{-9}$ & 5.00 \\
\hline $1.92 \times 10^{-3}$ & $1.33 \times 10^{-9}$ & 3.99 & $5.73 \times 10^{-11}$ & 5.00 & $1.40 \times 10^{-9}$ & 4.00 & $6.29 \times 10^{-11}$ & 5.00 \\
\hline
\end{tabular}

with $\left(\bar{x}_{1}, \bar{x}_{2}\right)=-(0.1,0.1), \epsilon=1 \times 1^{-2}$, and right-hand side $f$ computed accordingly. The choice $\boldsymbol{\kappa}=\boldsymbol{\kappa}_{1}$ corresponds to an isotropic problem and is used to assess the performance of the method in the simplest possible setting. The choice $\boldsymbol{\kappa}=\boldsymbol{\kappa}_{2}$, originally proposed by Le Potier [28], corresponds to an anisotropic, heterogeneous problem where the principal axes of the diffusion tensor vary at each point of the domain. For both choices, we solve the corresponding problem on both the triangular and (predominantly) hexagonal mesh families depicted in Figure 1 which correspond, respectively, to the mesh family 1 of the FVCA5 benchmark [24], and to the mesh used in the numerical examples of [19]. The convergence results are reported in Tables $3-6$. There, we show the history of convergence of $\left\|\boldsymbol{q}-\boldsymbol{q}_{h}\right\|$, see the label $H^{1}$, and of $\left\|u-u_{h}^{*}\right\|$, see the label $L^{2}$, and provide the 
TABLE 4. Hexagonal mesh family (isotropic homogeneous test case $\boldsymbol{\kappa}=\boldsymbol{\kappa}_{1}$ ).

\begin{tabular}{|c|c|c|c|c|c|c|c|c|}
\hline \multirow[b]{2}{*}{$h$} & \multicolumn{4}{|c|}{$\mathrm{HHO}(k)$} & \multicolumn{4}{|c|}{$\mathrm{HHO}(k+1)$} \\
\hline & $H^{1}$ & ECR & $L^{2}$ & $\mathrm{ECR}$ & $H^{1}$ & ECR & $L^{2}$ & ECR \\
\hline \multicolumn{9}{|c|}{$k=0$} \\
\hline $6.30 \times 10^{-2}$ & 0.16 & - & 0.18 & - & 0.16 & - & 0.25 & - \\
\hline $3.42 \times 10^{-2}$ & $8.83 \times 10^{-2}$ & 1.00 & $5.36 \times 10^{-2}$ & 2.01 & $8.98 \times 10^{-2}$ & 0.97 & $7.49 \times 10^{-2}$ & 1.95 \\
\hline $1.72 \times 10^{-2}$ & $3.68 \times 10^{-2}$ & 1.27 & $1.46 \times 10^{-2}$ & 1.89 & $3.72 \times 10^{-2}$ & 1.28 & $2.05 \times 10^{-2}$ & 1.89 \\
\hline $8.59 \times 10^{-3}$ & $1.38 \times 10^{-2}$ & 1.41 & $3.82 \times 10^{-3}$ & 1.93 & $1.40 \times 10^{-2}$ & 1.41 & $5.36 \times 10^{-3}$ & 1.93 \\
\hline $4.30 \times 10^{-3}$ & $5.00 \times 10^{-3}$ & 1.47 & $9.72 \times 10^{-4}$ & 1.98 & $5.04 \times 10^{-3}$ & 1.48 & $1.37 \times 10^{-3}$ & 1.97 \\
\hline \multicolumn{9}{|c|}{$k=1$} \\
\hline $6.30 \times 10^{-2}$ & $3.99 \times 10^{-2}$ & - & $3.27 \times 10^{-2}$ & - & $4.31 \times 10^{-2}$ & - & $3.66 \times 10^{-2}$ & - \\
\hline $3.42 \times 10^{-2}$ & $6.53 \times 10^{-3}$ & 2.96 & $4.59 \times 10^{-3}$ & 3.21 & $6.82 \times 10^{-3}$ & 3.02 & $4.74 \times 10^{-3}$ & 3.35 \\
\hline $1.72 \times 10^{-2}$ & $1.11 \times 10^{-3}$ & 2.58 & $6.11 \times 10^{-4}$ & 2.93 & $1.13 \times 10^{-3}$ & 2.62 & $6.12 \times 10^{-4}$ & 2.98 \\
\hline $8.59 \times 10^{-3}$ & $1.90 \times 10^{-4}$ & 2.54 & $7.85 \times 10^{-5}$ & 2.96 & $1.91 \times 10^{-4}$ & 2.56 & $7.75 \times 10^{-5}$ & 2.98 \\
\hline $4.30 \times 10^{-3}$ & $3.28 \times 10^{-5}$ & 2.54 & $9.93 \times 10^{-6}$ & 2.99 & $3.28 \times 10^{-5}$ & 2.55 & $9.74 \times 10^{-6}$ & 3.00 \\
\hline \multicolumn{9}{|c|}{$k=2$} \\
\hline $6.30 \times 10^{-2}$ & $3.26 \times 10^{-3}$ & - & $4.02 \times 10^{-3}$ & - & $3.26 \times 10^{-3}$ & - & $4.06 \times 10^{-3}$ & - \\
\hline $3.42 \times 10^{-2}$ & $4.35 \times 10^{-4}$ & 3.30 & $2.86 \times 10^{-4}$ & 4.33 & $4.31 \times 10^{-4}$ & 3.31 & $2.88 \times 10^{-4}$ & 4.33 \\
\hline $1.72 \times 10^{-2}$ & $5.15 \times 10^{-5}$ & 3.10 & $1.89 \times 10^{-5}$ & 3.95 & $5.14 \times 10^{-5}$ & 3.09 & $1.89 \times 10^{-5}$ & 3.96 \\
\hline $8.59 \times 10^{-3}$ & $6.20 \times 10^{-6}$ & 3.05 & $1.22 \times 10^{-6}$ & 3.95 & $6.19 \times 10^{-6}$ & 3.05 & $1.21 \times 10^{-6}$ & 3.96 \\
\hline $4.30 \times 10^{-3}$ & $7.61 \times 10^{-7}$ & 3.03 & $7.70 \times 10^{-8}$ & 3.99 & $7.60 \times 10^{-7}$ & 3.03 & $7.63 \times 10^{-8}$ & 3.99 \\
\hline \multicolumn{9}{|c|}{$k=3$} \\
\hline $6.30 \times 10^{-2}$ & $5.69 \times 10^{-4}$ & - & $4.26 \times 10^{-4}$ & - & $5.89 \times 10^{-4}$ & - & $4.42 \times 10^{-4}$ & - \\
\hline $3.42 \times 10^{-2}$ & $3.46 \times 10^{-5}$ & 4.58 & $1.73 \times 10^{-5}$ & 5.24 & $3.47 \times 10^{-5}$ & 4.64 & $1.74 \times 10^{-5}$ & 5.30 \\
\hline $1.72 \times 10^{-2}$ & $2.22 \times 10^{-6}$ & 4.00 & $5.90 \times 10^{-7}$ & 4.92 & $2.23 \times 10^{-6}$ & 3.99 & $5.89 \times 10^{-7}$ & 4.93 \\
\hline $8.59 \times 10^{-3}$ & $1.41 \times 10^{-7}$ & 3.97 & $1.91 \times 10^{-8}$ & 4.94 & $1.41 \times 10^{-7}$ & 3.98 & $1.90 \times 10^{-8}$ & 4.95 \\
\hline $4.30 \times 10^{-3}$ & $8.88 \times 10^{-9}$ & 4.00 & $6.09 \times 10^{-10}$ & 4.98 & $8.89 \times 10^{-9}$ & 3.99 & $6.05 \times 10^{-10}$ & 4.98 \\
\hline
\end{tabular}

TABle 5. Triangular mesh family (anisotropic, heterogeneous test case $\boldsymbol{\kappa}=\boldsymbol{\kappa}_{2}$ ).

\begin{tabular}{|c|c|c|c|c|c|c|c|c|}
\hline \multirow[b]{2}{*}{$h$} & \multicolumn{4}{|c|}{$\mathrm{HHO}(k)$} & \multicolumn{4}{|c|}{$\mathrm{HHO}(k+1)$} \\
\hline & $H^{1}$ & ECR & $L^{2}$ & ECR & $H^{1}$ & $\mathrm{ECR}$ & $L^{2}$ & ECR \\
\hline \multicolumn{9}{|c|}{$k=0$} \\
\hline $3.07 \times 10^{-2}$ & 0.14 & - & 0.11 & - & 0.14 & - & 0.15 & - \\
\hline $1.54 \times 10^{-2}$ & $7.06 \times 10^{-2}$ & 1.00 & $2.84 \times 10^{-2}$ & 2.01 & $7.04 \times 10^{-2}$ & 0.99 & $3.67 \times 10^{-2}$ & 2.00 \\
\hline $7.68 \times 10^{-3}$ & $3.53 \times 10^{-2}$ & 1.00 & $7.10 \times 10^{-3}$ & 1.99 & $3.53 \times 10^{-2}$ & 0.99 & $9.19 \times 10^{-3}$ & 1.99 \\
\hline $3.84 \times 10^{-3}$ & $1.77 \times 10^{-2}$ & 1.00 & $1.78 \times 10^{-3}$ & 2.00 & $1.77 \times 10^{-2}$ & 1.00 & $2.30 \times 10^{-3}$ & 2.00 \\
\hline $1.92 \times 10^{-3}$ & $8.83 \times 10^{-3}$ & 1.00 & $4.44 \times 10^{-4}$ & 2.00 & $8.83 \times 10^{-3}$ & 1.00 & $5.75 \times 10^{-4}$ & 2.00 \\
\hline \multicolumn{9}{|c|}{$k=1$} \\
\hline $3.07 \times 10^{-2}$ & $1.36 \times 10^{-2}$ & - & $1.16 \times 10^{-2}$ & - & $1.40 \times 10^{-2}$ & - & $1.21 \times 10^{-2}$ & - \\
\hline $1.54 \times 10^{-2}$ & $3.28 \times 10^{-3}$ & 2.06 & $1.46 \times 10^{-3}$ & 3.00 & $3.40 \times 10^{-3}$ & 2.05 & $1.52 \times 10^{-3}$ & 3.01 \\
\hline $7.68 \times 10^{-3}$ & $8.10 \times 10^{-4}$ & 2.01 & $1.83 \times 10^{-4}$ & 2.98 & $8.40 \times 10^{-4}$ & 2.01 & $1.90 \times 10^{-4}$ & 2.99 \\
\hline $3.84 \times 10^{-3}$ & $2.02 \times 10^{-4}$ & 2.00 & $2.28 \times 10^{-5}$ & 3.00 & $2.09 \times 10^{-4}$ & 2.01 & $2.37 \times 10^{-5}$ & 3.00 \\
\hline $1.92 \times 10^{-3}$ & $5.04 \times 10^{-5}$ & 2.00 & $2.86 \times 10^{-6}$ & 2.99 & $5.23 \times 10^{-5}$ & 2.00 & $2.97 \times 10^{-6}$ & 3.00 \\
\hline \multicolumn{9}{|c|}{$k=2$} \\
\hline $3.07 \times 10^{-2}$ & $1.01 \times 10^{-3}$ & - & $9.53 \times 10^{-4}$ & - & $1.04 \times 10^{-3}$ & - & $1.02 \times 10^{-3}$ & - \\
\hline $1.54 \times 10^{-2}$ & $1.22 \times 10^{-4}$ & 3.06 & $6.03 \times 10^{-5}$ & 4.00 & $1.27 \times 10^{-4}$ & 3.05 & $6.48 \times 10^{-5}$ & 4.00 \\
\hline $7.68 \times 10^{-3}$ & $1.50 \times 10^{-5}$ & 3.01 & $3.78 \times 10^{-6}$ & 3.98 & $1.57 \times 10^{-5}$ & 3.00 & $4.06 \times 10^{-6}$ & 3.98 \\
\hline $3.84 \times 10^{-3}$ & $1.87 \times 10^{-6}$ & 3.00 & $2.37 \times 10^{-7}$ & 4.00 & $1.95 \times 10^{-6}$ & 3.01 & $2.54 \times 10^{-7}$ & 4.00 \\
\hline $1.92 \times 10^{-3}$ & $2.33 \times 10^{-7}$ & 3.00 & $1.48 \times 10^{-8}$ & 4.00 & $2.42 \times 10^{-7}$ & 3.01 & $1.59 \times 10^{-8}$ & 4.00 \\
\hline \multicolumn{9}{|c|}{$k=3$} \\
\hline $3.07 \times 10^{-2}$ & $8.49 \times 10^{-5}$ & - & $5.91 \times 10^{-5}$ & - & $8.95 \times 10^{-5}$ & - & $6.49 \times 10^{-5}$ & - \\
\hline $1.54 \times 10^{-2}$ & $5.39 \times 10^{-6}$ & 4.00 & $1.87 \times 10^{-6}$ & 5.01 & $5.70 \times 10^{-6}$ & 3.99 & $2.05 \times 10^{-6}$ & 5.01 \\
\hline $7.68 \times 10^{-3}$ & $3.38 \times 10^{-7}$ & 3.98 & $5.85 \times 10^{-8}$ & 4.98 & $3.58 \times 10^{-7}$ & 3.98 & $6.41 \times 10^{-8}$ & 4.98 \\
\hline $3.84 \times 10^{-3}$ & $2.11 \times 10^{-8}$ & 4.00 & $1.83 \times 10^{-9}$ & 5.00 & $2.24 \times 10^{-8}$ & 4.00 & $2.01 \times 10^{-9}$ & 5.00 \\
\hline $1.92 \times 10^{-3}$ & $1.33 \times 10^{-9}$ & 3.99 & $5.73 \times 10^{-11}$ & 5.00 & $1.40 \times 10^{-9}$ & 4.00 & $6.29 \times 10^{-11}$ & 5.00 \\
\hline
\end{tabular}


TABLE 6. Hexagonal mesh family (anisotropic, heterogeneous test case $\boldsymbol{\kappa}=\boldsymbol{\kappa}_{2}$ ).

\begin{tabular}{|c|c|c|c|c|c|c|c|c|}
\hline \multirow[b]{2}{*}{$h$} & \multicolumn{4}{|c|}{$\mathrm{HHO}(k)$} & \multicolumn{4}{|c|}{$\operatorname{HHO}(k+1)$} \\
\hline & $H^{1}$ & ECR & $L^{2}$ & ECR & $H^{1}$ & ECR & $L^{2}$ & ECR \\
\hline \multicolumn{9}{|c|}{$k=0$} \\
\hline $6.30 \times 10^{-2}$ & 1.26 & - & 0.18 & - & 0.16 & - & 0.25 & - \\
\hline $3.42 \times 10^{-2}$ & 0.58 & 1.26 & $5.80 \times 10^{-2}$ & 1.86 & $8.98 \times 10^{-2}$ & 0.97 & $7.49 \times 10^{-2}$ & 1.95 \\
\hline $1.72 \times 10^{-2}$ & 0.22 & 1.40 & $1.84 \times 10^{-2}$ & 1.67 & $3.72 \times 10^{-2}$ & 1.28 & $2.05 \times 10^{-2}$ & 1.89 \\
\hline $8.59 \times 10^{-3}$ & $8.34 \times 10^{-2}$ & 1.41 & $4.98 \times 10^{-3}$ & 1.88 & $1.40 \times 10^{-2}$ & 1.41 & $5.36 \times 10^{-3}$ & 1.93 \\
\hline $4.30 \times 10^{-3}$ & $3.09 \times 10^{-2}$ & 1.43 & $1.35 \times 10^{-3}$ & 1.89 & $5.04 \times 10^{-3}$ & 1.48 & $1.37 \times 10^{-3}$ & 1.97 \\
\hline \multicolumn{9}{|c|}{$k=1$} \\
\hline $6.30 \times 1^{-2}$ & 0.15 & - & $2.56 \times 1^{-2}$ & $\overline{-}$ & $4.31 \times 1^{-2}$ & - & $3.66 \times 1^{-2}$ & - \\
\hline $3.42 \times 10^{-2}$ & $3.65 \times 10^{-2}$ & 2.30 & $4.98 \times 10^{-3}$ & 2.68 & $6.82 \times 1^{-3}$ & 3.02 & $4.74 \times 1^{-3}$ & 3.35 \\
\hline $1.72 \times 1^{-2}$ & $8.30 \times 1^{-3}$ & 2.15 & $6.68 \times 1^{-4}$ & 2.92 & $1.13 \times 10^{-3}$ & 2.62 & $6.12 \times 10^{-4}$ & 2.98 \\
\hline $8.59 \times 10^{-3}$ & $1.63 \times 10^{-3}$ & 2.34 & $8.54 \times 10^{-5}$ & 2.96 & $1.91 \times 10^{-4}$ & 2.56 & $7.75 \times 10^{-5}$ & 2.98 \\
\hline $4.30 \times 10^{-3}$ & $2.82 \times 10^{-4}$ & 2.54 & $1.09 \times 10^{-5}$ & 2.97 & $3.28 \times 1^{-5}$ & 2.55 & $9.74 \times 1^{-6}$ & 3.00 \\
\hline \multicolumn{9}{|c|}{$k=2$} \\
\hline $6.30 \times 1^{-2}$ & $2.13 \times 1^{-2}$ & - & $4.02 \times 1^{-3}$ & - & $3.26 \times 10^{-3}$ & - & $4.06 \times 10^{-3}$ & - \\
\hline $3.42 \times 10^{-2}$ & $2.09 \times 10^{-3}$ & 3.80 & $3.09 \times 10^{-4}$ & 4.20 & $4.31 \times 10^{-4}$ & 3.31 & $2.88 \times 10^{-4}$ & 4.33 \\
\hline $1.72 \times 1^{-2}$ & $2.08 \times 1^{-4}$ & 3.36 & $2.15 \times 1^{-5}$ & 3.88 & $5.14 \times 10^{-5}$ & 3.09 & $1.89 \times 10^{-5}$ & 3.96 \\
\hline $8.59 \times 10^{-3}$ & $2.26 \times 10^{-5}$ & 3.20 & $1.40 \times 10^{-6}$ & 3.93 & $6.19 \times 10^{-6}$ & 3.05 & $1.21 \times 10^{-6}$ & 3.96 \\
\hline $4.30 \times 1^{-3}$ & $2.55 \times 1^{-6}$ & 3.15 & $8.91 \times 1^{-8}$ & 3.98 & $7.60 \times 10^{-7}$ & 3.03 & $7.63 \times 10^{-8}$ & 3.99 \\
\hline \multicolumn{9}{|c|}{$k=3$} \\
\hline $6.30 \times 10^{-2}$ & $4.59 \times 10^{-3}$ & - & $4.29 \times 10^{-4}$ & - & $5.89 \times 10^{-4}$ & - & $4.42 \times 10^{-4}$ & - \\
\hline $3.42 \times 10^{-2}$ & $1.43 \times 10^{-4}$ & 5.68 & $1.70 \times 10^{-5}$ & 5.28 & $3.47 \times 10^{-5}$ & 4.64 & $1.74 \times 10^{-5}$ & 5.30 \\
\hline $1.72 \times 10^{-2}$ & $8.77 \times 10^{-6}$ & 4.06 & $6.01 \times 10^{-7}$ & 4.86 & $2.23 \times 10^{-6}$ & 3.99 & $5.89 \times 10^{-7}$ & 4.93 \\
\hline $8.59 \times 10^{-3}$ & $4.82 \times 10^{-7}$ & 4.18 & $1.95 \times 10^{-8}$ & 4.94 & $1.41 \times 10^{-7}$ & 3.98 & $1.90 \times 10^{-8}$ & 4.95 \\
\hline $4.30 \times 10^{-3}$ & $2.52 \times 10^{-8}$ & 4.26 & $6.13 \times 10^{-10}$ & 5.00 & $8.89 \times 10^{-9}$ & 3.99 & $6.05 \times 10^{-10}$ & 4.98 \\
\hline
\end{tabular}

corresponding estimated convergence rate (ECR). The error is normalized with respect to the corresponding norm of the exact solution. We can observe that both methods yield very similar results for the homogeneous isotropic test case ( $c f$. Tabs. 3 and 4 ), whereas a clear advantage of the $\operatorname{HHO}(k+1)$ method is observed in the anisotropic, heterogeneous test case ( $c f$. Tabs. 5 and 6$)$ when it comes to the $H^{1}$ norm. The difference is less pronounced on the hexagonal mesh than on the triangular mesh.

\section{CONCluding REMARKS}

We have established a bridge between the HHO method and the general framework of HDG methods where the key step has been the identification of the numerical trace associated with HHO methods. The bridge between HHO and HDG methods has allowed us to incorporate into the HDG methods the new, subtle way of defining the numerical trace for the flux in HHO methods. The present approach can be carried out for many other partial differential equations for which HDG methods have been already defined.

Acknowledgements. The first author would like to thank Guosheng Fu for fruitful discussions concerning the numerical traces of the HHO and HDG methods considered here. The third author acknowledges partial support from CEA/DAM.

\section{REFERENCES}

[1] T. Arbogast, M.F. Wheeler and I. Yotov, Mixed finite elements for elliptic problems with tensor coefficients as cell-centered finite differences. SIAM J. Numer. Anal. 34 (1997) 828-852.

[2] B. Ayuso de Dios, K. Lipnikov and G. Manzini, The nonconforming virtual element method. Preprint arxiv:1405.3741 (2014).

[3] L. Beirão da Veiga, K. Lipnikov and G. Manzini, The Mimetic Finite Difference Method for Elliptic Problems. MSEA. Springer (2014).

[4] J. Bonelle and A. Ern, Analysis of compatible discrete operator schemes for elliptic problems on polyhedral meshes. ESAIM: M2AN 48 (2014) 553-581. 
[5] F. Brezzi, K. Lipnikov and M. Shashkov, Convergence of the mimetic finite difference method for diffusion problems on polyhedral meshes. SIAM J. Numer. Anal. 43 (2005) 1872-1896.

[6] F. Brezzi, K. Lipnikov, M. Shashkov and V. Simoncini, A new discretization methodology for diffusion problems on generalized polyhedral meshes. Comput. Methods Appl. Mech. Engrg. 196 (2007) 3682-3692.

[7] P. Castillo, B. Cockburn, I. Perugia and D. Schötzau, An a priori error analysis of the local discontinuous Galerkin method for elliptic problems. SIAM J. Numer. Anal. 38 (2000) 1676-1706.

[8] Z. Chen, BDM mixed methods for a nonlinear elliptic problem. J. Comput. Appl. Math. 53 (1994) $207-223$.

[9] B. Cockburn, J. Gopalakrishnan and R. Lazarov, Unified hybridization of discontinuous Galerkin, mixed, and continuous Galerkin methods for second order elliptic problems. SIAM J. Numer. Anal. 47 (2009) 1319-1365.

[10] B. Cockburn, J. Gopalakrishnan and F.-J. Sayas, A projection-based error analysis of HDG methods. Math. Comput. 79 (2010) 1351-1367.

[11] B. Cockburn, W. Qiu and K. Shi, Conditions for superconvergence of HDG methods for second-order elliptic problems. Math. Comput. 81 (2012) 1327-1353.

[12] B. Cockburn and K. Shi, Devising HDG methods for Stokes flow: An overview. Comput. Fluids 98 (2014) $221-229$.

[13] D.A. Di Pietro, J. Droniou and A. Ern, A discontinuous-skeletal method for advection-diffusion-reaction on general meshes. SIAM J. Numer. Anal. 53 (2015) 2135-2157.

[14] D.A. Di Pietro and A. Ern, Mathematical Aspects of Discontinuous Galerkin Methods. Vol. 69 of Math. Appl. Springer-Verlag, Berlin (2012).

[15] D.A. Di Pietro and A. Ern, Equilibrated tractions for the Hybrid High-Order method. C. R. Acad. Sci Paris, Ser. I 353 (2015) 279-282.

[16] D.A. Di Pietro and A. Ern, A hybrid high-order locking-free method for linear elasticity on general meshes. Comput. Meth. Appl. Mech. Engrg. 2832015 1-21.

[17] D.A. Di Pietro and A. Ern, Hybrid high-order methods for variable-diffusion problems on general meshes. C. R. Acad. Sci Paris, Ser. I 353 (2015) 31-34.

[18] D.A. Di Pietro, A. Ern and S. Lemaire. An arbitrary-order and compact-stencil discretization of diffusion on general meshes based on local reconstruction operators. Comput. Meth. Appl. Math. 14 (2014) 461-472.

[19] D.A. Di Pietro and S. Lemaire, An extension of the Crouzeix-Raviart space to general meshes with application to quasiincompressible linear elasticity and Stokes flow. Math. Comput. 84 (2015) 1-31.

[20] J. Droniou and R. Eymard, A mixed finite volume scheme for anisotropic diffusion problems on any grid. Numer. Math. 105 (2006) 35-71.

[21] J. Droniou, R. Eymard, T. Gallouët and R. Herbin, A unified approach to mimetic finite difference, hybrid finite volume and mixed finite volume methods. M3AS Math. Models Methods Appl. Sci. 20 (2010) 1-31.

[22] T. Dupont and R. Scott, Polynomial approximation of functions in Sobolev spaces. Math. Comput. 34 (1980) $441-463$.

[23] R. Eymard, T. Gallouët and R. Herbin, Discretization of heterogeneous and anisotropic diffusion problems on general nonconforming meshes. SUSHI: a scheme using stabilization and hybrid interfaces. IMA J. Numer. Anal. 30 (2010) $1009-1043$.

[24] R. Herbin and F. Hubert, Benchmark on Discretization Schemes for Anisotropic Diffusion Problems on General Grids. In Finite Volumes for Complex Applications V. Edited by R. Eymard and J.-M. Hérard. John Wiley and Sons (2008) 659-692.

[25] H. Kabaria, A. Lew and B. Cockburn, A hybridizable discontinuous Galerkin formulation for nonlinear elasticity. Comput. Methods Appl. Mech. Engrg. 283 (2015) 303-329.

[26] J. Koebbe, A computationally efficient modification of mixed finite element methods for flow problems with full transmissivity tensors. Numer. Methods Partial Differ. Equ. 9 (1993) 339-355.

[27] Y. Kuznetsov, K. Lipnikov and M. Shashkov. Mimetic finite difference method on polygonal meshes for diffusion-type problems. Comput. Geosci. 8 (2004) 301-324.

[28] C. Le Potier, A finite Volume Method for the Approximation of Highly Anisotropic Diffusion Operators on Unstructured Meshes. In Finite Volumes for Complex Applications IV (2005).

[29] C. Lehrenfeld, Hybrid Discontinuous Galerkin methods for incompressible flow problems. Diploma thesis, MathCCES/IGPM, RWTH Aachen (2010).

[30] K. Lipnikov and G. Manzini. A high-order mimetic method on unstructured polyhedral meshes for the diffusion equation. $J$. Comput. Phys. 272 (2014) 360-385.

[31] I. Oikawa, A hybridized discontinuous Galerkin method with reduced stabilization. J. Sci. Comput. 65 (2015) $327-340$.

[32] W. Qiu and K. Shi, An HDG method for linear elasticity with strongly symmetric stresses. Preprint arXiv:1312.1407 (2015).

[33] W. Qiu and K. Shi. An HDG method for convection-diffusion equations. J. Sci. Comput. 66 (2016) $346-357$.

[34] S.-C. Soon, Hybridizable discontinuous Galerkin methods for solid mechanics. Ph.D. thesis, University of Minnesota, Minneapolis (2008).

[35] S.-C. Soon, B. Cockburn and H.K. Stolarski, A hybridizable discontinuous Galerkin method for linear elasticity. Int. J. Numer. Methods Engrg. 80 (2009) 1058-1092. 